\title{
Physical Sensors Based on Laser-Induced Graphene: A Review
}

\author{
Altynay Kaidarova, and Jürgen Kosel
}

\begin{abstract}
Physical sensors form the fundamental building blocks of a multitude of advanced applications that detect and monitor the surroundings and communicate the acquired physical data. The everlasting need for more compliant, low-cost, and energy-efficient sensor solutions has led to considerable interest in enhancing their features and operation limits even further. While graphene has emerged as a promising candidate material, due to its outstanding electrical and mechanical properties, it is still not available in large volumes for practical applications. Meanwhile, Laser-Induced Graphene has opened new perspectives for a versatile, durable, printed physical sensing platform capable of detecting various physical parameters across a range of conditions and subjects. In this review, LIG physical sensors were categorized into four broad types based on their transduction mechanism: mechanical, thermal, magnetic, and electromagnetic. We summaries various design strategies established for preparing reliable physical sensors without the involvement of chemical treatments, synthesis, and multi-step fabrication processes. The review considers the effects of laser choice, lasing environment, and parameters on graphene properties. We also discuss a broad spectrum of applications of LIG physical sensors in fields ranging from healthcare, tactile sensing, environmental monitoring, energy harvesting, and soft robotics to desalination and THz modulation.
\end{abstract}

Index Terms — flexible, graphene, lightweight, laser-induced graphene, physical sensors.

\section{Introduction}

$\mathrm{P}$ hyscal sensors have established an efficient connection with the real-world, continuously monitoring and controlling various complex environments on a daily basis, from local temperature and material deformation to health parameters[1]. Physical variables, such as pressure, temperature, humidity, strain, bending or electromagnetic field, are also of high relevance for a multitude of advanced sensing applications such as in distributed diagnostics [2], robotics [3-5], electronic skins [6-8], functional clothing[9-11], and many other Internet-ofThings solutions [9, 12-14]. With rapidly developing needs in these areas, there is an increasing demand for effective implementation of such sensors. Recent years have seen the advent of a particular class of physical sensors, which are lightweight, flexible and wearable devices featuring high degrees of deformability, conformability, long-term stability, increased sensitivity, transparency, and biocompatibility [1, 15$18]$.

Multifunctional graphene has been recently identified as a promising sensor material, due to its outstanding mechanical, electrical, and thermal properties [19-23]. Notably, the graphene-based 3D porous macrostructures, like foams, sponges, aerogels, and hydrogels, were reported to provide stability for physical sensors during repeated loading and unloading cycles [24-26]. Currently, methods for producing graphene can broadly be classified into two different categories: bottom-up and top-down. The bottom-up approach uses chemistry to assemble carbon atoms creating a monolayer

A. Kaidarova is with the Computer, Electrical and Mathematical Sciences \& Engineering (CEMSE) department, King Abdullah University of Science and Technology (KAUST), 23955, Thuwal, Kingdom of Saudi Arabia 2395 (altynay.kaidarova@kaust.edu.sa). structure. The primary technique used in this process is chemical vapor deposition [27-29], which allows the production of a monolayer of graphene directly onto a copper $[28,30]$ or nickel substrate $[31,32]$. However, transferring graphene onto flexible substrates often induces defects, cracks, and wrinkles, causing a drop in the overall quality and reduction in the graphene properties [33-35]. The top-down approach involves breaking millions of stacked layers of graphite into graphene monolayers. This was first achieved using sticky tape, which exfoliated graphite until a single layer of graphene was obtained [36]. Such an approach is not suitable for large scale production of graphene. The liquid phase exfoliation is another method that places graphite into a solvent, which is shocked vigorously using sonication points, resulting in graphite exfoliating spontaneously into the solvent [37-39]. This method produces a graphene solution and upon the solvent's evaporation - graphene powder. As it stands, with this technique only grams of graphene can be obtained, which is not an adequate amount for use in industry projects or composite materials.

In 2014 a multifunctional 3D graphene was obtained via direct laser writing on commercial polyimide in ambient atmosphere conditions [40]. This method combines large-area graphene preparation and patterning with a single fabrication step without the need for expensive cleanroom equipment, wet chemical steps, reducing agents, solvents, subsequent treatments, or other supporting processes. This laser-induced graphene (LIG) has stimulated research in many areas, ranging from fundamental to applied sciences, investigating the laser graphitization

J.Kosel is with the Sensor Systems Division, Silicon Austria Labs, Austria; and with the Computer, Electrical and Mathematical Sciences \& Engineering (CEMSE) Division, King Abdullah University of Science and Technology (KAUST), 23955, Thuwal, Kingdom of Saudi Arabia 2395 (jurgen.kosel@kaust.edu.sa). 

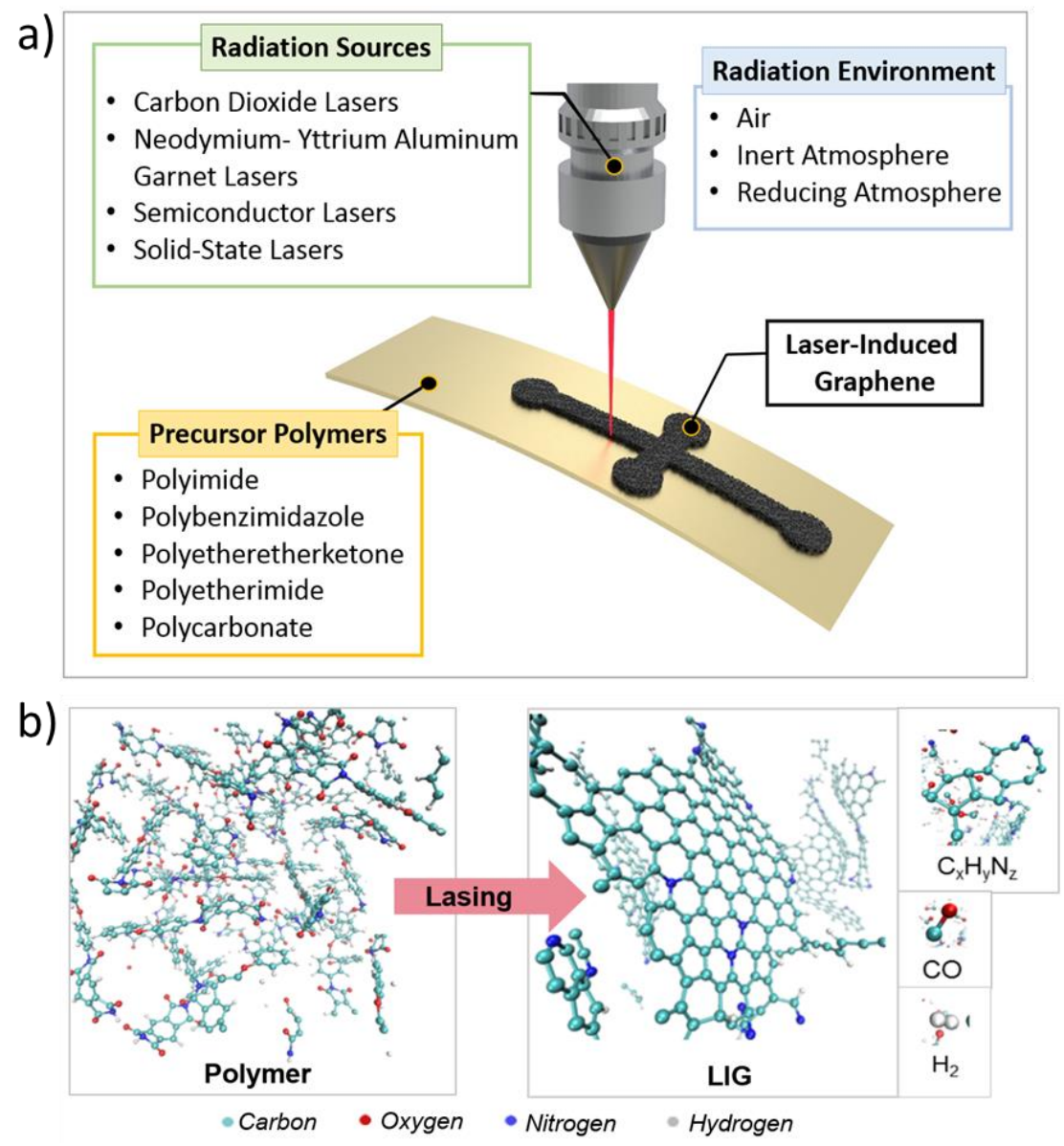

Fig. 1. a) Fabrication of LIG using various laser types, polymers, and laser radiation environments. b). Formation of LIG from polymer accompanied by the decomposition of species, such as $\mathrm{CO}, \mathrm{H}_{2}$, and $\mathrm{C}_{\mathrm{x}} \mathrm{H}_{\mathrm{y}} \mathrm{N}_{\mathrm{z}}$ [41].

process [41-43], effects of various lasers [44-46], environments [47, 48], and lasing parameters [49-51], to the development of a large variety of flexible physical and chemical sensors. The latter utilizes LIG as an electrochemical transducer while incorporating additional chemical recognition systems and surface functionalization to interact with analytic molecules [52-54], such as glucose[55], tyrosine [56], dopamine [57], bisphenol [53], thrombin [58], ascorbic and uric acid [56]. The majority of the existing review articles [59-62] has mainly focused on LIG-based energy storage devices with electrodeposition [52, 63-65], an active catalyst with heteroatom doping [66-68], photodetectors with photosensitized materials integration [69], and biosensors with surface functionalization [70-72], all of which benefit from the high surface area of LIG and improved facilitation of electron transfer. While others focused more on reviewing laser-assisted processing of CVD graphene and its derivatives [73-76].

This review focuses on developments that utilized LIG to implement physical sensors without additional chemical treatments and functionalization processes. The LIG physical transducers were divided into four groups based on their signals and applications, which are mechanical, thermal, magnetic, and electromagnetic. We discuss the 3D graphene formation process and the tuning of its characteristics via laser parameters, surrounding environment, and features of various lasers. The review offers insights into different applications and the challenges faced as well as opportunities provided by the development of flexible and wearable physical sensors based on LIG.

\section{FORMATION OF POROUS GRAPHENE}

\section{A. Transformation process}

The polymer is converted to a porous graphene film through laser photothermal (long laser wavelength, >400 nm) or photochemical (short laser wavelength, $<400 \mathrm{~nm}$ ) processes associated with two consecutive steps: carbonization and graphitization [60]. The former produces amorphous tetrahedral carbon, also known as diamond-like carbon. The latter leads to rapid decomposition of gaseous products, the transformation of the existing $\mathrm{sp}^{3}$ bonds to $\mathrm{sp}^{2}$ bonds along with crystallization of the amorphous matrix, and an increase in electrical conductivity [77].

Various laser types, substrate materials, and environmental conditions have been utilized to obtain porous graphene, which are listed in Fig.1a and discussed in more detail in section IIIA. The optical propagation of the laser within the polymer films plays a decisive role in regulating the composition of the synthesized graphene. The actual laser intensity after entering the polymer is given by

$$
I=I_{o} e^{-\alpha / z}
$$

Polymers that have been used to form LIG include, but are not 

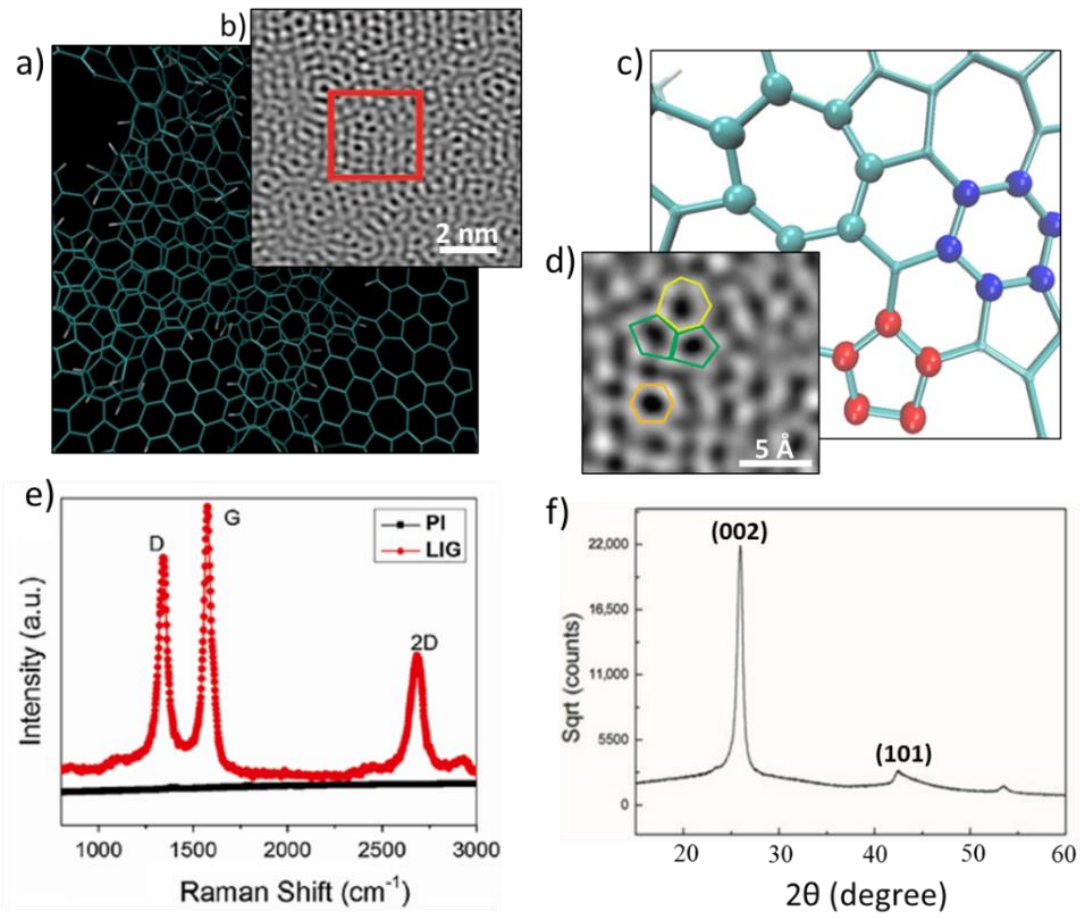

Fig. 2. a) The polycrystalline nature of LIG with grain boundary obtained through simulation and b) through TEM image (inset); c) Simulated LIG surface, (d) TEM image showing the existence of different types of rings (5-, 6- and 7-member rings) [40, 41]. e) Typical Raman spectra of LIG with prominent 2D peak [42] and f) XRD with a sharp (002) peak [84].

limited to Polyimide (PI) [40], Polybenzimidazole (PBM) [78], Polyether ether ketone (PEEK) [79], Polyetherimide (PEI) [80], an aromatic ring in their molecular structure composed of Polycarbonate (PC) [81] as seen in Fig.1a. These polymers have carbon $(\mathrm{C})$ and hydrogen $(\mathrm{H})$, as well as nitrogen $(\mathrm{N})$, and Oxygen $(\mathrm{O})$ atoms. The molecular differences in the polymer precursor affect the gases evolved during LIG formation. The general reaction observed during the LIG creation is summarized in Fig. 1b [41]. Typically, the base polymer is heated through a laser, producing LIG and predominantly $\mathrm{CO}$ and $\mathrm{H}_{2}$ gases along with hydrocarbon $\left(\mathrm{C}_{\mathrm{x}} \mathrm{H}_{\mathrm{y}} \mathrm{N}_{\mathrm{z}}\right)$ species.

Considering the most commonly used combination of a $\mathrm{CO}_{2}$ laser and PI, carbonization is reported to commence at $673 \mathrm{~K}$, while graphitization occurs upon further heating of the PI to at least $773 \mathrm{~K}$ [77]. The gases produced from LIG formation are removed from the system after the course of $1.25 \mathrm{~ns}$ [41]. During the initial $0.2 \mathrm{~ns}$ of lasing, an amorphous structure is formed that grows into an ordered graphene structure with 6membered carbon rings (Fig. 2a) [41]. This is in agreement with the results presented by Lin et al. [40], where TEM demonstrated the ordered ring structure of graphene formed on the PI (Fig. 2b). Both studies have also reported unusual structures containing 5- and 7-member rings, which is a result of steric stresses, the rapid formation and cooling of the graphene and trapping it in a higher energy state (Fig. 2c, d)[40, 41]. LIG formation has been generally confirmed via Raman and X-Ray Diffraction (XRD) analyses. The prominent 2D peak
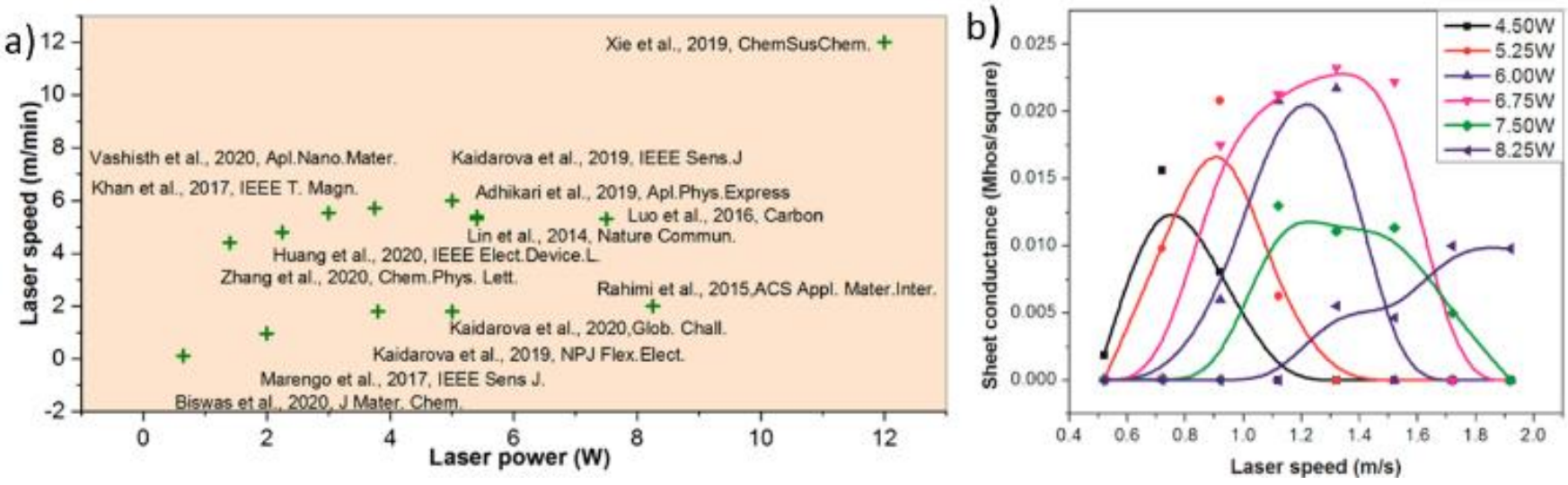

Fig. 3. a) Overview of typically reported $\mathrm{CO}_{2}$ laser parameters (power and speed) to fabricate porous graphene on PI. b) Sheet conductance of carbon trace on PI as a function of laser fabrication parameters [99] 
centered around $2700 \mathrm{~cm}^{-1}$ in the Raman spectrum of LIG (Fig. 2e) $[42,59,82]$ was identified to be the same as in singlelayer graphene, but with a larger width at half maximum of around $60 \mathrm{~cm}^{-1}$ [83]. Meanwhile, the sharp peak observed around $26^{\circ}(002)$ in the XRD spectrum suggested the formation of a highly crystalline graphene structure (Fig.2f) [84]. The computer-controlled lasers allow printing arbitrary structures of customizable shapes and sizes for macro- and micro-manufacturing. The lasing parameters need to be carefully considered due to their profound impact on the resultant morphology of porous graphene [50, 85]. These lasing parameters include power $(\mathrm{W})$, scanning speed $(\mathrm{m} / \mathrm{min})$, pulses per inch (PPI), and operational distance, $\mathrm{z}(\mathrm{mm})$. Power refers to the magnitude of the energy with which the laser nozzle operates. Speed refers to the rate of movement of the laser nozzle over the sample in $\mathrm{x}-\mathrm{y}$ directions. The $\mathrm{z}$-axis defines the focal point of the laser on the polymer $[42,86]$

\section{B. Effect of laser parameters}

An overview of the combinations of $\mathrm{CO}_{2}$ laser power levels and speeds that have been employed to fabricate LIG is provided in Fig.3a. As can be seen, a wide range of parameter sets can be used, as long as the threshold energy is reached [99]. In the case of PI, threshold energy of $7.9 \pm 0.6 \mathrm{Jcm}^{-2}$ is needed to form LIG [77], which is in the range of previously reported energy estimations [51, 59, 99].

Laser parameters also affect the sheet resistance, conductivity, crystallite size, thickness, and wettability of porous graphene. For instance, depending on the power applied, the sheet resistance and conductivity of LIG (obtained from $\mathrm{CO}_{2}$ laser ablation of PI) can be in the range of $5-115 \Omega \mathrm{sq}^{-1}$ and 500 $2500 \mathrm{Sm}^{-1}$, respectively $[51,100]$. An excessive laser power or speed, nevertheless, lead to a decrease in conductance, due to an increase in defect level of the graphitic tracks or oxidation of the graphene, as seen in Fig. 3b [99]. The amount of laser power can also affect the thickness, line width and crystallinity of LIG (obtained from $\mathrm{CO}_{2}$ laser ablation of PI). The thickness can be tuned in the range from $20 \mu \mathrm{m}$ to $100 \mu \mathrm{m}$ [42], line width can be in the range of $110 \mu \mathrm{m}$ to $240 \mu \mathrm{m}$ [86] with crystalline size varying from $20 \mathrm{~nm}$ to $40 \mathrm{~nm}[40,77]$.

The wettability of the LIG was previously controlled via scanning speed and the frequency of the laser incident radiation [50]. A superhydrophobic behavior of porous graphene with contact angles of $>150^{\circ}$ has been reported when laser scanning speed and frequency exceeded $12 \mathrm{~m} / \mathrm{min}$ and $20 \mathrm{kHz}$, respectively. These laser parameters induced a different structure rearrangement and gas evolution in PI, affecting its conduction properties [50]. This observation was in line with the wettability results of carbon-based materials, which were highly amplified by the nano-morphology of the LIG foams [113].

\section{AdDITIONAL TUNING OPTIONS}

\section{A. Choice of laser}

Table I summarizes the lasers that have been utilized for obtaining 3D porous graphene from PI.

1) Infrared laser. The gas discharge of $\mathrm{CO}_{2}$ molecules can effectively produce large laser energy at IR wavelength (10.6 $\mu \mathrm{m}, 943.40 \mathrm{~cm}^{-1}$ ). The IR laser couples to $\mathrm{C}-\mathrm{C}$ bonds present in the polymer and provides efficient photothermal heating [114]. The rastering capabilities of this laser enable the patterning of LIG into any desired geometry. Since polymers possess a large absorption at $10.6 \mu \mathrm{m}$, the carbonization process starts as soon as the photon enters it. This results in the topmost layers of the precursor to turning into graphene, leaving the bottom part pristine to function as mechanical support. Biswas et al. have thoroughly investigated the transformation of PI into LIG using a $\mathrm{CO}_{2}$ laser. The carbonization of PI occurred at a threshold power of $(0.21 \pm 0.02) \mathrm{W}$ and a fluence of $(3.31 \pm 0.32)$ $\mathrm{J} \mathrm{cm}^{-2}$, while graphitization was reported at a power of $(0.50$ $\pm 0.03) \mathrm{W}$ and a fluence of $(7.88 \pm 0.47) \mathrm{J} \mathrm{cm}^{-2}$ [77]. The threshold power shows a linear dependence on the laser speed, i.e., a higher speed requires a higher power in order to initiate the graphitization [46]. Meanwhile, near IR lasers, such as Nd:

Table I. Overview of the lasers utilized for LIG fabrication

\begin{tabular}{|c|c|c|c|c|c|c|c|c|}
\hline $\begin{array}{l}\text { Optical } \\
\text { region }\end{array}$ & Type & Wavelength & Manufacturer & Model & $\begin{array}{l}\text { Power } \\
\text { (W) }\end{array}$ & $\begin{array}{c}\text { Threshold } \\
\text { Fluence \& Power }\end{array}$ & Ref. & $\begin{array}{c}\text { Spot } \\
\text { size }(\mu \mathrm{m})\end{array}$ \\
\hline \multirow{7}{*}{ IR } & \multirow{6}{*}{$\mathrm{CO}_{2}$} & \multirow{6}{*}{$10.6 \mu \mathrm{m}$} & \multirow{5}{*}{$\begin{array}{l}\text { Universals } \\
\text { Laser } \\
\text { Systems }\end{array}$} & PLS 6.75 & $10-75$ & \multirow{7}{*}{$\begin{array}{c}(0.50 \pm 0.03) \mathrm{W} \\
(7.88 \pm 0.47) \mathrm{J} \mathrm{cm}^{-2}\end{array}$} & [87-98] & \multirow{7}{*}{$\sim 60-240$} \\
\hline & & & & PLS 6MW & $10-75$ & & [99] & \\
\hline & & & & XLS10MWH & $10-150$ & & [100-102] & \\
\hline & & & & VL-300 & $30-50$ & & [86] & \\
\hline & & & & VLS 2.3 & $10-30$ & & {$[85,103-106]$} & \\
\hline & & & Coryart & $\begin{array}{l}\text { Microlaser } \\
\quad \text { C40 }\end{array}$ & 40 & & [107] & \\
\hline & Nd:YAG & $1064 \mathrm{~nm}$ & DMG Mori & Lasertec 40 & 100 & & {$[46]$} & \\
\hline \multirow{4}{*}{$\begin{array}{l}\text { Visible } \\
\text { (blue- } \\
\text { violet) }\end{array}$} & \multirow{4}{*}{$\begin{array}{l}\text { Laser } \\
\text { Diode }\end{array}$} & $450 \mathrm{~nm}$ & QSRAM & & 1.6 & \multirow{4}{*}{$\begin{array}{c}0.161 \mathrm{~W} \\
83.4 \mathrm{~J} \mathrm{~cm}^{-2}\end{array}$} & [108] & \multirow{4}{*}{$>12$} \\
\hline & & & Nano Pro III & & $0.5-5$ & & [109] & \\
\hline & & $405 \mathrm{~nm}$ & Q-BAIHE & $\begin{array}{c}405 \mathrm{ML}- \\
300 \mathrm{e} 2290\end{array}$ & $0.015-0.3$ & & [110] & \\
\hline & & & Laser Co. & $\begin{array}{l}\text { M-33A405- } \\
500-G, 91\end{array}$ & 0.5 & & {$[45,48,111]$} & \\
\hline UV & $\mathrm{Nd}: \mathrm{YVO}_{4}$ & $355 \mathrm{~nm}$ & Inngu Laser & & $<40$ & $\begin{array}{l}\sim 0.3 \mathrm{~W} \\
\sim 15 \mathrm{~J} \mathrm{~cm}^{-2}\end{array}$ & {$[44,112]$} & $\sim 30-60$ \\
\hline
\end{tabular}


YAG $(1064 \mathrm{~nm})$, can be easily integrated into a chamber with environment control and can be equipped with a fast galvoscanning system with a nanosecond pulse, for rapid prototyping and large scale production. However, no graphitization has been observed when the laser energy was low or under defocusing conditions on the PI films. Due to the spot size of the beam and diffraction limitations, infrared lasers typically produce $60-100 \mu \mathrm{m}$ line widths [115].

2) UV lasers. The energy of photons increases with decreasing wavelengths. When the wavelength of external light approaches the UV region (typically smaller than $400 \mathrm{~nm}$ ), the photochemical effects significantly influence the synthesis of graphene by removing the oxygen residuals on the graphene skeleton [60]. Differing from the photothermal effect, the absorbed photon energy can directly break the chemical bonding, especially when the photon energy is larger than the dissociation energy [112]. Initially applied for laser reduction of graphene oxide [116], UV lasers (with threshold power of $15 \mathrm{~kW} \mathrm{~cm}^{-2}$ ) are currently used for direct writing of graphene to avoid significant heating of the PI. Because of the smaller wavelengths, UV lasers can be also focused to smaller spot sizes than IR lasers. Carvalho et al. reported a LIG strain sensor produced with a UV laser using the power of $0.3 \mathrm{~W}$ and a fluence of $15 \mathrm{~J} \mathrm{~cm}^{-2}$ [44]. The smallest LIG resolution formed directly from UV lasing were in the range of $30-50 \mu \mathrm{m}$, while the thickness was in the range of $\sim 5 \mu \mathrm{m}[44,111]$.

3) Visible blue-violet lasers. An improved resolution has been recently demonstrated using visible $405 \mathrm{~nm}$ laser, which produced the smallest feature of $\sim 12 \mu \mathrm{m}$ [111]. Stanford et al. demonstrated a strong absorption of visible blue-violet light photons, therefore making visible light lasers attractive for photothermally converting PI to LIG. The laser enabled the near-surface formation of LIG, which resulted in LIG thicknesses of $<5 \mu \mathrm{m}$ [111], comparable to LIG formed from a UV laser. A threshold energy of $83.4 \mathrm{~J} \mathrm{~cm}^{-2}$ and power of $0.161 \mathrm{~W}$ were required to form LIG from a single pulse laser exposure . As can be noticed from Table I, visible lasers also utilize significantly lower powers, which could ultimately be attractive for the commercialization of LIG devices.

\section{B. Influence of Lasing Environment}

The lasing environment influences the pore size, conductivity, and thickness of the LIG, as well as hydrophobicity and hydrophilicity of the LIG surfaces [47, 48, 119].

Cai et al. have investigated the graphitization process under laser irradiation in air and inert atmospheres [48]. When the laser writing process was conducted in air, the graphitization process was accompanied by an oxidation process, which resulted in the formation of thicker graphene films with rough surface morphology. The LIG pores were reported to be micrometer-sized and broadly distributed with high oxygen content [48]. In contrast, when the laser writing process was carried out in an inert atmosphere, such as Ar, the oxidation process was suppressed, and the laser carbonization effect dominated the process. Inert environment facilitated the formation of thinner LIG films with smooth surface morphology, relatively narrow pore size distribution, and high carbon content and conductivity $[48,120]$. The change in the surrounding gas atmosphere also allowed a tremendous change in the water contact angle on the LIG, from $0^{\circ}$ (superhydrophilic), when using $\mathrm{O}_{2}$ or air, to $>160^{\circ}$ (superhydrophobic), when using inert or reducing gases (Ar, $\mathrm{H}_{2}$, or $\mathrm{SF}_{6}$ ) [47]. Li et al. showed that different wetting properties are due to the changes in surface morphology and surface chemistry of LIG structures. The fabrication in a controlled gas atmosphere mimics the traditional CVD process for the growth and modification of graphene properties but

Table II. Summary of LIG physical sensors applications.

\begin{tabular}{|c|c|c|c|c|c|c|}
\hline \multirow[b]{2}{*}{ Applied Fields } & \multirow[b]{2}{*}{ Applications } & \multirow[b]{2}{*}{ Ref. } & \multicolumn{4}{|c|}{ Sensor type } \\
\hline & & & Mechanical & Thermal & Magnetic & $\begin{array}{l}\text { Electro- } \\
\text { magnetic }\end{array}$ \\
\hline \multirow{6}{*}{$\begin{array}{l}\text { Healthcare, } \\
\text { (Humans and/or } \\
\text { Animals) }\end{array}$} & Heart-rate/Pulse monitoring & {$[44,89,104,117]$} & $\checkmark$ & $\checkmark$ & & \\
\hline & Gesture recognition & {$[86,89,104]$} & $\checkmark$ & $\checkmark$ & & \\
\hline & Artificial throat & {$[96,108,117]$} & & $\checkmark$ & & \\
\hline & Artificial muscle & {$[104]$} & & $\checkmark$ & & \\
\hline & Speed detection & {$[89,91]$} & $\checkmark$ & & $\checkmark$ & \\
\hline & Plantar pressure measurements & {$[90]$} & $\checkmark$ & & & \\
\hline \multirow{5}{*}{$\begin{array}{l}\text { Environmental } \\
\text { monitoring }\end{array}$} & Temperature detection & {$[88,110,118]$} & & $\checkmark$ & & \\
\hline & Gas sensing & {$[98]$} & & $\checkmark$ & & \\
\hline & Pressure sensing & {$[90,94]$} & $\checkmark$ & & & \\
\hline & Flow measurements & {$[89,118]$} & $\checkmark$ & & $\checkmark$ & \\
\hline & Humidity sensing & {$[107]$} & & & & $\checkmark$ \\
\hline \multirow{4}{*}{ Robotics } & Tactile sensing & {$[90,94]$} & $\checkmark$ & & & \\
\hline & Gesture-based control & {$[86,89,94,104]$} & $\checkmark$ & $\checkmark$ & & \\
\hline & Sound detecting & {$[96,108,117]$} & & $\checkmark$ & & \\
\hline & Proximity \& Positioning & {$[91,106]$} & & & $\checkmark$ & \\
\hline \multirow{5}{*}{$\begin{array}{l}\text { Manufacturing/ } \\
\text { Quality Check }\end{array}$} & Localized heating & [93] & & & & $\checkmark$ \\
\hline & Sterilizing \& Filtering & [102] & & $\checkmark$ & & \\
\hline & Seawater desalination & [46] & & & & $\checkmark$ \\
\hline & Energy harvesting & {$[100]$} & $\checkmark$ & & & \\
\hline & Imaging Security & [109] & & & & $\checkmark$ \\
\hline
\end{tabular}


replaces the high-temperature oven heating by laser irradiation [121].

\section{Physical SENSORS MADE OF LIG}

In this section, we review the advances of LIG physical sensors by dividing them into four major groups, which are mechanical, thermal, magnetic and electromagnetic. The broad spectrum of applications of these sensors in the fields such as healthcare, environmental monitoring, robotics, manufacturing, and quality control/manufacturing, are summarized in Table II. In particular, working mechanisms and device structures of various LIG transducers are discussed.

\section{A. Mechanical transducers}

Mechanical transducers detect some form of mechanical deformation and translate it into an electrical signal. The mechanical deformation can occur due to a variety of stimuli, such as changes in human physiological movement, gas/liquid flow, or pressure fluctuations [122]. LIG mechanical sensors and transducers have been developed for tactile sensing, energy harvesting, and underwater monitoring applications. These developments are classified according to their working mechanisms, which are piezoresistive, capacitive, and triboelectric. Table III summarizes various parameters of LIG mechanical transducers obtained from the laser ablation of PI in ambient conditions. The aspects of the fabrication process, such as the radiation source, geometrical design of the sensor, and lasing parameters, have shown a cumulative effect on the resultant performance of the sensors. It should be noted that, although PI serves as mechanically flexible support, it is not easily stretched (2.5 GPa[123]) and must be modified to enable large elastic strains (>2\%). This is especially important when a sensor has to conform to the very elastic surface of human skins.

Piezoresistive transducers. The piezoresistance of LIG electrodes printed on flexible PI films has been exploited and utilized in a range of configurations, including measurements of strain, deflection, curvature, force, and pressure $[86,89,90$, $94,97,103,105]$. The high variation of the electrical resistance of multilayer samples under strain is induced by the displacement of the layers and changes of their overlapping area. The absolute values of the resistance and its change can be tailored by the number of turns and dimensions of the meander-shaped LIG piezoresistive electrode [97].

Kaidarova et al. utilized LIG on a flexible PI film to transduce a signal via cantilever deflection, which was supported at one end with the other end free (Fig. 4a). An external force, $F$, applied to the tip of the cantilever induced uniaxial stress described by (2).

$$
\sigma=\frac{F(L-d) c}{I_{A}}
$$

where $L$ is the cantilever length, $c$ is the distance to the neutral axis, $d$ is the distance from the support, and $I_{A}$ is the area moment of inertia [89]. The longitudinal stress varies linearly through the cantilever thickness and along its length. The maximum stress, $\sigma_{\max }$, occurs at the surface of the cantilever at the anchor point, as shown in Fig. 4a. The interaction of a sensor with an external force, such as water flow, along the $y$-axis,
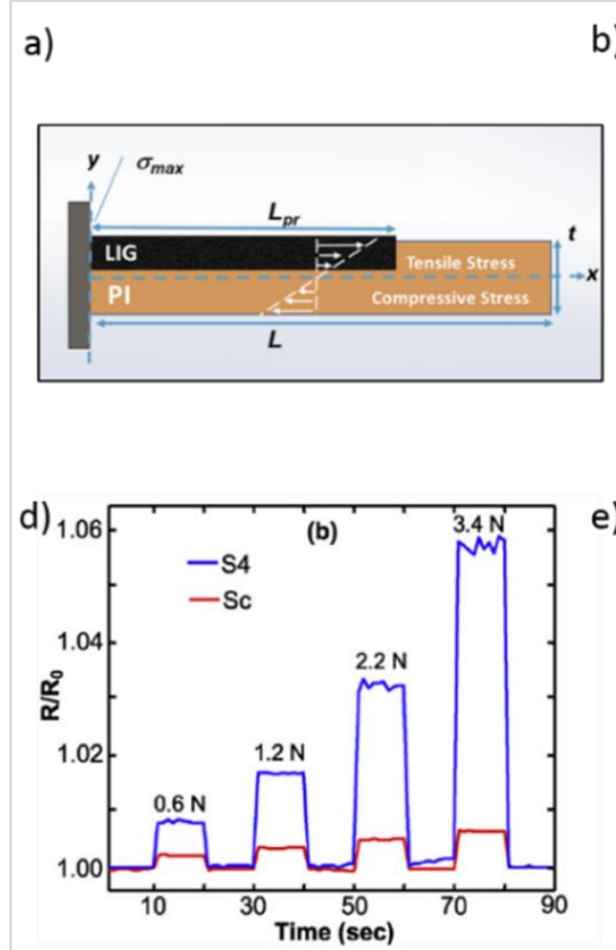

b)

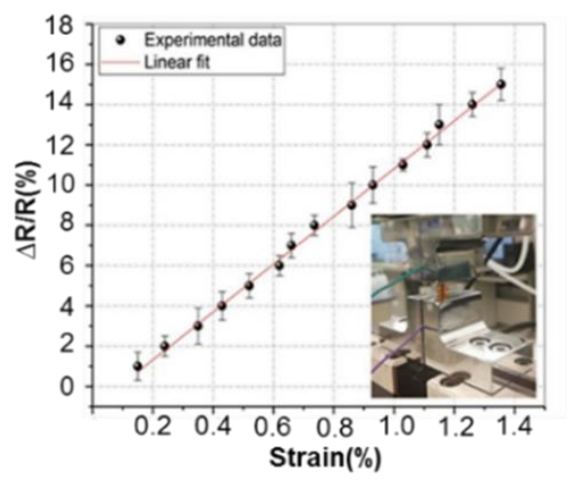

e)

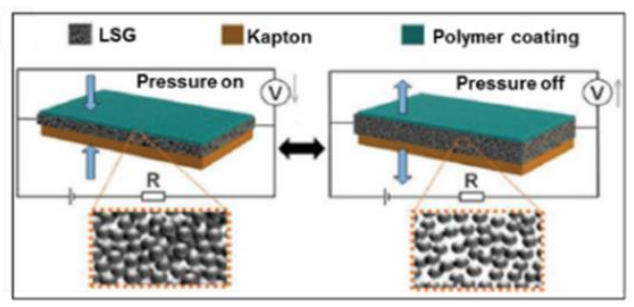

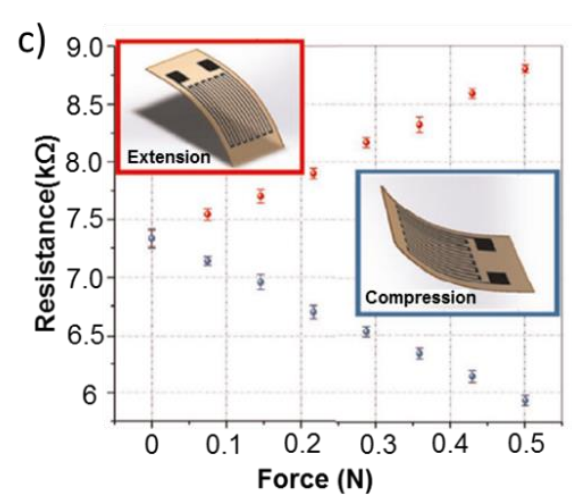

f)

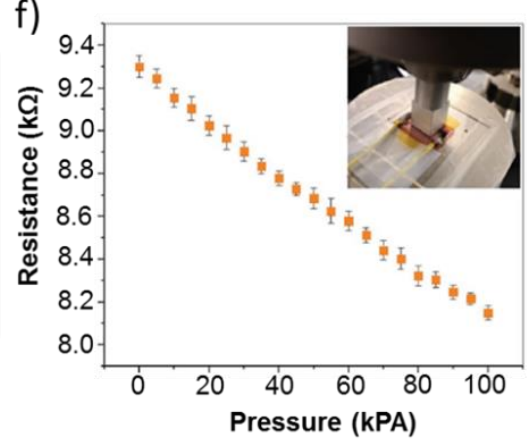

Fig. 4. Schematic cross-section of the piezoresistive cantilever beam [89]. b) Strain-induced resistance change of LIG [89]. c) Resistance as a function of a force induced during extension and compression of LIG [89]. d) Resistance change for LIG (S4) and commercial sensors ( $\mathrm{S}_{c}$ ) at different compressive loads [86]. e) Operation principle of pressure-sensitive LIG sensors [90]. f) Average resistance of the LIG pressure sensor as a result of consecutive linear loading cycles [90]. 
induces cantilever bending and frictional resistance change. Fig. $4 \mathrm{~b}$ shows a linear relationship between strain and relative change in resistance of the LIG. A gauge factor $(\mathrm{GF}) \simeq 11.2$ (Young modulus $\simeq 2.1 \mathrm{GPa}$, yield strength $\simeq 83 \mathrm{MPa}$ ) was extracted from the slope (Fig. 4b), which is in the range of the values of metal strain gauges [89]. Luo et al. reported that the GF could be further increased by increasing the ratio of power over scanning speed, reaching a GF of 110 [86]. The electrical resistance of the LIG strain sensor linearly increases with tension and decreases with compression, as shown in Fig. 4c [89]. Fig. $4 d$ compares the performance of commercial $\left(\mathrm{S}_{\mathrm{c}}\right)$ and LIG strain $\left(\mathrm{S}_{4}\right)$ sensors of the same geometry subjected to compression. The resistance change of the LIG sensor was 11 times more than that of a commercial sensor $(350 \Omega$, Cat \# SGD-5/350-LY41, Omega) after being bent under a 3.4 $\mathrm{N}$ load. Former increased by $\sim 5.6 \%$, while the latter increased only by $\sim 0.5 \%$ [86].

The piezoresistive effect of 3D graphene was also utilized to develop pressure sensors that feature high resolution with a detection limit of $10 \mathrm{~Pa}$ and an extremely wide dynamic range of at least $20 \mathrm{MPa}$ [90]. The decrease in resistance with the applied pressure was explained by the establishment of a larger number of conductive paths inside of the graphene structure. Thereby, the porous LIG recovers to its initial state after releasing the pressure. (Fig. 4 e-f).

Piezoresistive LIG sensors were utilized in various applications, such as marine animals' speed monitoring, current flow velocity measurements in a coral reef, pressure sensing at an underwater depth of $2 \mathrm{~km}$ in highly saline water, human joint bending, motion tracking, heart rate monitoring, plantar pressure measurements, and tactile sensing applications [44, $86,89,90]$.
Capacitive transducers. Capacitive LIG sensors are realized by an interdigitated capacitive (IDC) electrode structure. The sensing mechanism is based on the dependency of the sensor capacitance on the sensor geometry (length (L), width (W), and the gap between the interdigitated fingers (d)) [124]. Elongated or shorted dimensions of the IDC-LIG senor change the gap between the fingers in either direction (Fig.5a), and hence its overall capacitance. It should be noted that such deformations also affect the distribution of the graphene over the substrate, which can contribute to the change in the resistance of the sensor. The impedance (Z) of LIG-IDC sensors under different loading conditions has been reported by Han et al. [94]. As Fig. 5 b shows, the impedance of the sensor increases, when the bending radius decreases. This is attributed to the increase in the gap between the interdigitated fingers, leading to a decrease of capacitance. Fig. 5c shows a linear increase in the sensor impedance with applied strain and a GF of 0.47 , which is considerably lower than the ones obtained using the piezoresistive effect. The IDC LIG sensor was deployed for monitoring of human joint movements and tactile sensing [124].

Triboelectric transducers. To convert wasted mechanical energy into electrical energy, Stanford et al. utilized LIG as a triboelectric material in a standard dielectric-to-conductor setup [100, 125].

The triboelectric nanogenerator (TENG) consisted of PI as a dielectric layer and aluminum (Al) as a positively charged conductor material, as shown in Fig.5d. When the PI was brought into contact with the $\mathrm{Al}$, contact electrification occurred as charges exchanged at the interface. When the layers got separated, the electrical potential caused current to flow
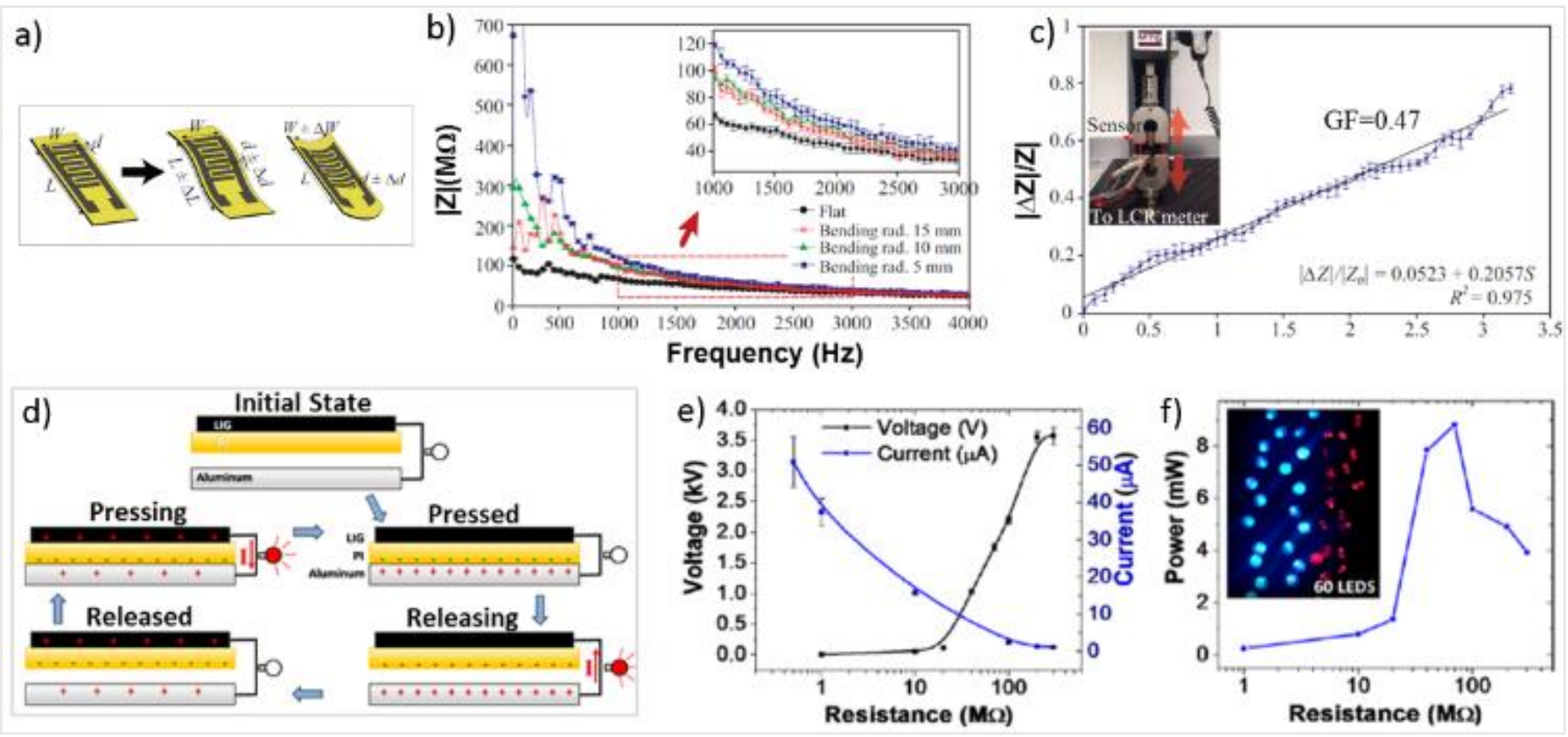

Fig. 5. a) Strain-induced deformations of IDC LIG sensors [94]. b) The response of the sensor to different bending radii of curvature [94]. c) Change in sensor impedance as a function of strain [94]. d) Mechanism for power generation by dielectric-to-conductor TENG using a LIG/PI film [100]. e) $V_{O C}$ and $I_{S C}$ as a function of the load resistance [100]. f) Power output by the device at different load resistances. The inset image shows LEDs powered by TENG [100]. 
Table III. Fabrication and performance parameters of mechanical transducers made of LIG.

\begin{tabular}{|c|c|c|c|c|c|c|c|c|}
\hline $\begin{array}{c}\text { Transduction } \\
\text { Type }\end{array}$ & $\begin{array}{l}\text { Mech. } \\
\text { Stimulus }\end{array}$ & $\begin{array}{l}\text { Radiation } \\
\text { Source }\end{array}$ & $\begin{array}{c}\text { Lasing } \\
\text { power(W), } \\
\operatorname{speed}(\mathrm{m} / \mathrm{min})\end{array}$ & $\begin{array}{c}\text { Geometry } \\
\text { Width(mm), Length(mm), } \\
\text { Number of turns }\end{array}$ & $\begin{array}{c}\text { GF } \\
\text { /Sensitivity }\end{array}$ & LOD & $\begin{array}{l}\text { Strain } \\
\text { /Dynamic } \\
\text { Range }\end{array}$ & Ref. \\
\hline \multirow{9}{*}{$\begin{array}{l}\text { Piezo- } \\
\text { resistive }\end{array}$} & \multirow{3}{*}{ Strain } & \multirow[t]{2}{*}{$\mathrm{IR}, \mathrm{CO}_{2}$} & $3.6,1.8$ & Meander, 7, 7,11 & 11.2 & - & $\begin{array}{l}1.4 \% \\
\text { strain }\end{array}$ & [89] \\
\hline & & & $4.5,3.8$ & Meander, 3.2, 24, 5 & 41.8 & - & $\begin{array}{l}1.5 \% \\
\text { strain }\end{array}$ & [86] \\
\hline & & $\mathrm{IR}, \mathrm{CO}_{2}$ & $1.32,4.44$ & Shutter, 10, 20 & 2.5 & - & $1.5 \%$ strain & [117] \\
\hline & \multirow[t]{2}{*}{ Force } & \multirow[b]{2}{*}{$\mathrm{IR}, \mathrm{CO}_{2}$} & $3.6,1.8$ & Meander, 7, 7.6,11 & $2.98 \Omega / \mathrm{mN}$ & - & - & [89] \\
\hline & & & $4.5,3.8$ & Meander, 3.2, 24,5 & $0.016 / \mathrm{N}$ & - & - & {$[86]$} \\
\hline & Deflection & $\mathrm{IR}, \mathrm{CO}_{2}$ & $3.6,1.8$ & Meander, $7,7.6,11$ & $0.21 \Omega / \mu \mathrm{m}$ & - & & [89] \\
\hline & \multirow{2}{*}{$\begin{array}{l}\text { Flow } \\
\text { (water) }\end{array}$} & \multirow[b]{2}{*}{$\mathrm{IR}, \mathrm{CO}_{2}$} & $3.6,1.8$ & Meander, 3.2, 24, 5 & $100 \Omega /(\mathrm{m} / \mathrm{s})$ & - & $3 \mathrm{~m} / \mathrm{s}$ & [89] \\
\hline & & & 2,1 & Meander, 10, 25, 3 & $20 \Omega /(\mathrm{m} / \mathrm{s})$ & - & $1 \mathrm{~m} / \mathrm{s}$ & [118] \\
\hline & Pressure & $\mathrm{IR}, \mathrm{CO}_{2}$ & 5,2 & Meander, $9.8,10,13$ & $\begin{array}{c}1.23 \cdot 10^{-3} \\
\mathrm{kPa}\end{array}$ & $10 \mathrm{~Pa}$ & $20 \mathrm{MPa}$ & {$[90]$} \\
\hline
\end{tabular}

from the triboelectrically positive $\mathrm{Al}$ to the LIG electrode through an external load via electrostatic induction [100]. Repeated contact and separation between the two electrodes of the TENG generated AC power. Fig. 5e shows the average voltages and currents generated as a function of the load resistance. A maximum open-circuit voltage of $3.5 \mathrm{kV}$ and a short-circuit current of $60 \mu \mathrm{A}$ were found. The peak power output was approximately $8.5 \mathrm{~mW}\left(\sim 2.4 \mathrm{Wm}^{-2}\right)$ at a resistance of $70 \mathrm{M} \Omega$, as seen in Fig.5e. The TENG simultaneously powered 60 blue and red light-emitting diodes in series (inset of Fig.5f). The TENG was also used to generate power from human movement by inserting it into the shoe sole of a flip-flop [100]. A rigid and thick Al incorporated into the TENG introduced a limitation to the overall flexibility of the system, while imposing additional and undesirable weight.

\section{B. Thermal transducers}

Electrothermal transducers. The response of a LIG sensor to a thermal stimulus was investigated by Bobinger et al.[110]. To convert the changes in resistance, due to temperature, into a voltage change, an excitation current of $0.59 \mathrm{~mA}$ was applied. The resistance of LIG showed a linear decrease with increasing temperature. Approximately $10 \%$ decrease in resistance was recorded, when the temperature was raised from 25 to $200^{\circ} \mathrm{C}$ [110]. The obtained temperature coefficient $(\alpha=-0.46 \pm 0.046$ $\mathrm{m}^{\circ} \mathrm{C}^{-1}$ ) was comparable with polycrystalline graphite below a temperature of $1400^{\circ} \mathrm{C}\left(\alpha=0.52 \mathrm{~m}^{\circ} \mathrm{C}^{-1}\right)[110,127]$ and graphene $\left(\alpha=0.5 \mathrm{~m}^{\circ} \mathrm{C}^{-1}\right)$ [128]. These results are in line with PDMS coated LIG thermistors characterized in seawater environment in the range of 18 to $40^{\circ} \mathrm{C}$, where a thermal coefficient of $\alpha=-$ $0.5 \mathrm{~m}^{\circ} \mathrm{C}^{-1}$ was reported [88]. The laser scribing process allowed tuning the geometry of the thermistor and incorporating it as a part of a miniaturized conductivity, temperature, and depth tag [87, 88, 129].

Flexible heaters based on the Joule effect have attracted great research interests, due to their wide applications ranging from gas sensors to self-sterilizing filters. LIG heaters with low working voltage, high steady-state temperature, fast response, and excellent thermal stability have been reported. Since the temperature for the decomposition of LIG and PI in air was reported to be $\sim 660^{\circ} \mathrm{C}$, and $580^{\circ} \mathrm{C}$, respectively, the maximum Joule-heating temperature of the heater was limited by the PI [102]. The temperature of the LIG heater reported by Bobinger et al. increased at a rate of $131 \pm 5{ }^{\circ} \mathrm{C} \mathrm{cm} / \mathrm{W}$, reaching a maximum operating temperature of $400^{\circ} \mathrm{C}$ [110]. The response of the LIG heater to extensive bending cycles, where the sample was bent to a minimum diameter of around $4 \mathrm{~mm}$ while subjected to electrical power of $1 \mathrm{~W}$, is shown in Fig. 6a, indicating good robustness against deformation [110]. Meanwhile, Huan et al. have improved the electrothermal performance of LIG heaters through a repeated laser writing process [130]. After the device was irradiated four times by the laser, it showed increased sensitivity of $189^{\circ} \mathrm{C} \mathrm{cm}^{2} / \mathrm{W}$ [130]

Self-sterilizing filter. A thermally actuated LIG membrane was used to capture and destroy microorganisms, bacteria, and molecules that can cause adverse biological reactions and diseases [102]. The decomposition of microorganisms occurred through a periodic Joule-heating mechanism, during which the LIG filter readily reached $>300{ }^{\circ} \mathrm{C}$. The filter was realized by lasing PI on both sides. An alternating hexagonal pattern of PI was left unused to reinforce the filtering process [102]. Two different morphologies of LIG were obtained by tuning the laser parameters. The outer surface served to capture larger particles and aerosols, which was a vertically grown forest of LIG with a height of $1 \mathrm{~mm}$, also known as LIG fibers (LIGF) [51, 131]. The center of the filter was composed of LIG (with pore sizes of 2.86-8.94 $\mathrm{nm}$ ) that captured smaller contaminants.

When LIG is subjected to airflow, microorganisms get trapped inside of it. The LIG filter is then Joule-heated, using DC power, for sterilization purposes. The experiments were carried out by mounting a LIG filter on a vacuum filtration system and 
backing it by a pore test polyethersulfone (PES) membrane, as shown in Fig. 6b [102]. The PES could capture contaminants in case any substance passed through the LIG filter. The optical densities of filters after exposing LIG and PES filters to culture media are shown in Fig.6c. The bacterial growth was observed in both filters as expected. The bacteria growth was high on the LIG filter and a PES reference filter, while it was low on the PES filter that followed the LIG filtration. This indicated that the LIG filter successfully prevented bacteria from reaching the PES filter. Meanwhile, there was no bacterial growth on the Joule-heated LIG filters, showing that Joule-heating at $300{ }^{\circ} \mathrm{C}$ successfully destroyed the bacteria [102]. However, the filtration efficiency was limited by a long surface residence time of adsorbents, reaching a few hours even under highvacuum conditions This was recently improved by Huang et al., who reported $99.998 \%$ filtration efficiency attained within $10 \mathrm{~min}$ under $0.75 \mathrm{~kW} / \mathrm{m}^{2}$ irradiation [132]. Since it was discovered that the SARS-CoV could be deactivated at $56{ }^{\circ} \mathrm{C}$ in $15 \mathrm{~min}$ [133], Huang et al. have taken advantage of the broadband absorption properties of LIG, and achieved this temperature by exposing LIG to sunlight. The health protection capacity of commercial face masks (activated carbon fiber (ACF) and melt-blown fabrics (MBF)) and LIG masks (Fig.6d) have been also evaluated. The surface temperature of LIG increased from 25 to $52{ }^{\circ} \mathrm{C}$ within $30 \mathrm{~s}$ and was maintained at $\sim 62{ }^{\circ} \mathrm{C}$ after further exposure to sunlight (Fig.6e). Meanwhile, the surface temperature of ACF was steady at $\sim 52{ }^{\circ} \mathrm{C}$ after $30 \mathrm{~s}$ of continuous irradiation, which is $10^{\circ} \mathrm{C}$ lower than that of LIG.
The lower temperature of ACF was explained by relatively large pores compared to LIG. The MBF was only $35^{\circ} \mathrm{C}$ even after the 60 s or longer irradiation. The bactericidal efficiency of LIG vastly improved to 99.84 and $99.998 \%$ after a 5 and 10 min exposure to sunlight, respectively (Fig. 6f). Meanwhile, ACF and MBF could only destroy $\sim 65$ and $85 \%$ of the bacteria after 10 min illumination and the number of bacteria remained substantial [132].

Gas sensors. A LIG sensor was developed to measure surrounding gases exploiting their thermal conductivity [98]. The transducer consisted of LIG electrodes that were connected by a $57 \mu \mathrm{m}$ wide LIG channel, as shown in Fig. 7a When a voltage was applied across the LIG, Joule heating was localized around the narrow channel, due to its large resistance. The heat transfer to its surroundings occurred via convective heat loss by Newton's law as given by

$$
\mathrm{q}=\mathrm{hA}\left(T_{a}-\mathrm{T}_{b}\right)
$$

where $q$ is the heat transferred, $h$ is the heat transfer coefficient, $A$ is the surface area of the LIG, $T_{a}$ and $T_{b}$ are the temperature of gas molecules and LIG channel, respectively [134]. The heat transfer coefficient, $h$, is dependent on the material parameters, such as thermal conductivity, viscosity, and heat capacity. When exposed to the gas, the temperature of the LIG channel was reduced, which resulted in increased resistance. Fig. 7b shows the response of the LIG sensor when exposed to $\mathrm{O}_{2}$ gas vs. vacuum, operating at $\sim 28 \mathrm{~mW}$. The response was repeatable, with no variation in the sensitivity.

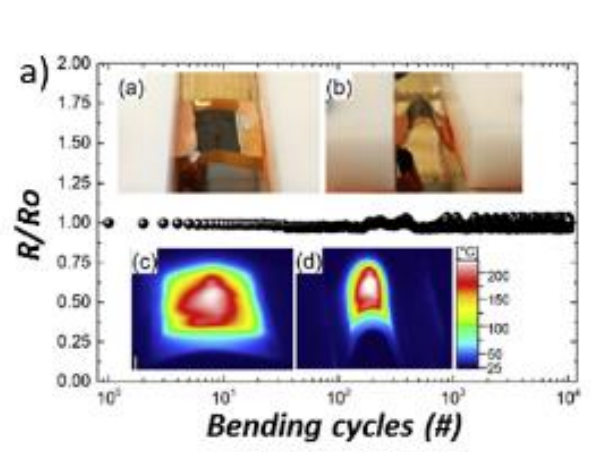

d)

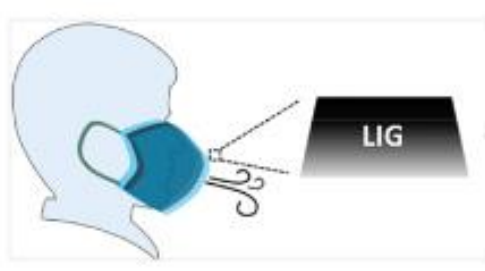

b)

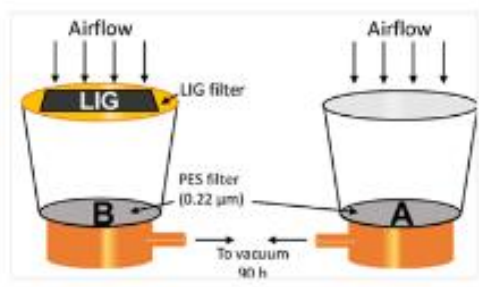

e)

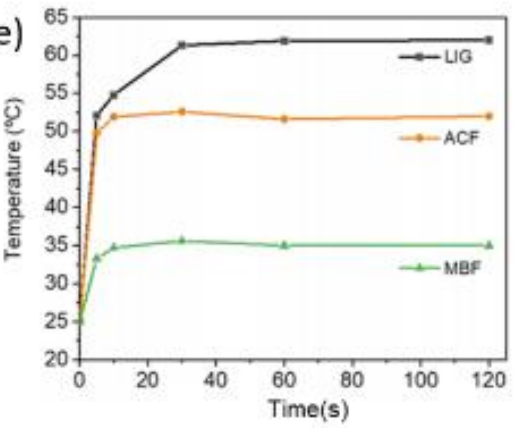

c)
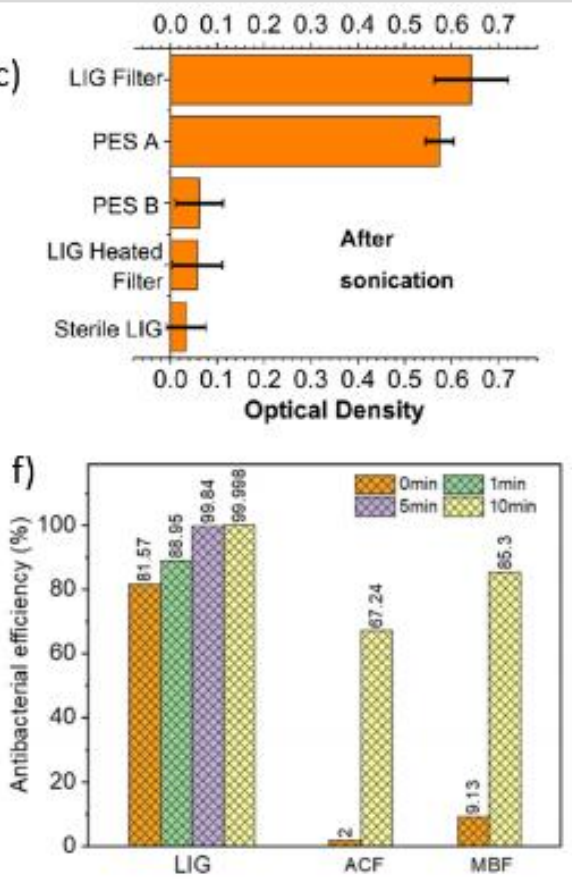

Fig.6. a) Normalized resistance of a LIG heater with respect to the increasing number of bending cycles [110]. The insets show optical images and infrared images of the heater in relaxed and bent positions. b) Schematic of the filtering-sterilization experimental setup [102]. c) The optical density of the filter after 24 hours incubation of bacteria [102]. d) Antibacterial LIG mask [132]. e) Temporal dependence of surface temperature under $0.75 \mathrm{~kW} / \mathrm{m}^{2}$ sunlight [133]. f) Antibacterial efficiency under different light exposure time [132]. 
The sensor also exhibited sensitivity to different gases, such as Ar, $\mathrm{He}, \mathrm{O}_{2}, \mathrm{CO}_{2}, \mathrm{~N}_{2}$, and $\mathrm{H}_{2}$. The highest sensitivity was reported for He, which had a high thermal conductivity (180 $\mathrm{mW} \mathrm{m}^{-1} \mathrm{~K}^{-1}$ ), while the sensitivity was lower for gases such as Ar that had a lower relative thermal conductivity $\left(25 \mathrm{~mW} \mathrm{~m}^{-1}\right.$ $\mathrm{K}^{-1}$ ). This indicates that the extent of cooling of the LIG filament depends on the thermal conductivity of the gas species. The sensor effectively detected subtle gas concentration changes, while demonstrating high reliability and repeatability [98]. LIG based gas sensors $\left(\mathrm{H}_{2}, \mathrm{NO}_{2}\right)$ were also realized using specific [135] and non-specific binding of chemicals [136]. For example, a linear sensing response to $\mathrm{H}_{2}$ concentrations was achieved via a biomimetic artificial nose with a turbinate-like structure [72], but that is beyond the scope of this review.

Electrothermal actuation. Soft electrothermal actuators based on PDMS coated LIG have been reported by Ling et al. [104] LIG served as a heater to introduce temperature differences via joule heating. The stress caused by the thermal expansion difference between PI and PDMS could deform 2D structures into 3D architectures via global and local bending. More than 20 architectures of various complex geometries were modeled by finite element analysis (FEA) [104]. Fig. 7c shows the fabrication process of a U-shaped electrothermal actuator, where thermal stress caused its bending towards the PI side. The bending angles were controlled by actuation voltages, which determined the temperature differences induced by LIG heaters. LIG actuators achieved the largest bending angle of $1080^{\circ}$ and a bending curvature of $3.3 \mathrm{~cm}^{-1}$ at an actuation voltage of $30 \mathrm{~V}$. The LIG actuators could bend and unbend for over 1000 times without degradation in performance [104].

It should be noted that, the actuator design with uniform thickness and cuts could only enable a global bending, as shown in Fig.7d. To achieve local bending of the actuator Fig. 7e, the authors used origami (i.e., thickness control) and kirigami (i.e., cutting patterns control) designs. In particular, a $\mathrm{CO}_{2}$ laser was used to thin and narrow the actuator on selected locations and create creases for folding. The actuator was utilized to control the 3D assembly of a flower-like structure via electromyogram (EMG) signals of various human gestures and to measure electrocardiogram (ECG) signals via a robotic finger (Fig. $7 \mathrm{f} \mathrm{-}$ g). The on-demand reversibility, long-term stability, and repeatability of LIG soft actuators still need to be systematically investigated.

Thermoacoustic transducers. Benefiting from the high thermal conductivity, low heat capacity, and high electrical conductivity of LIG, Tao et al. developed an artificial throat that is capable of functioning as a thermoacoustic sound source and detector [108] (Fig. 8a). To this end, the LIG artificial throat was clamped under a microphone. Applying AC voltage to the LIG device induced periodic Joule heating and the expansion of air,

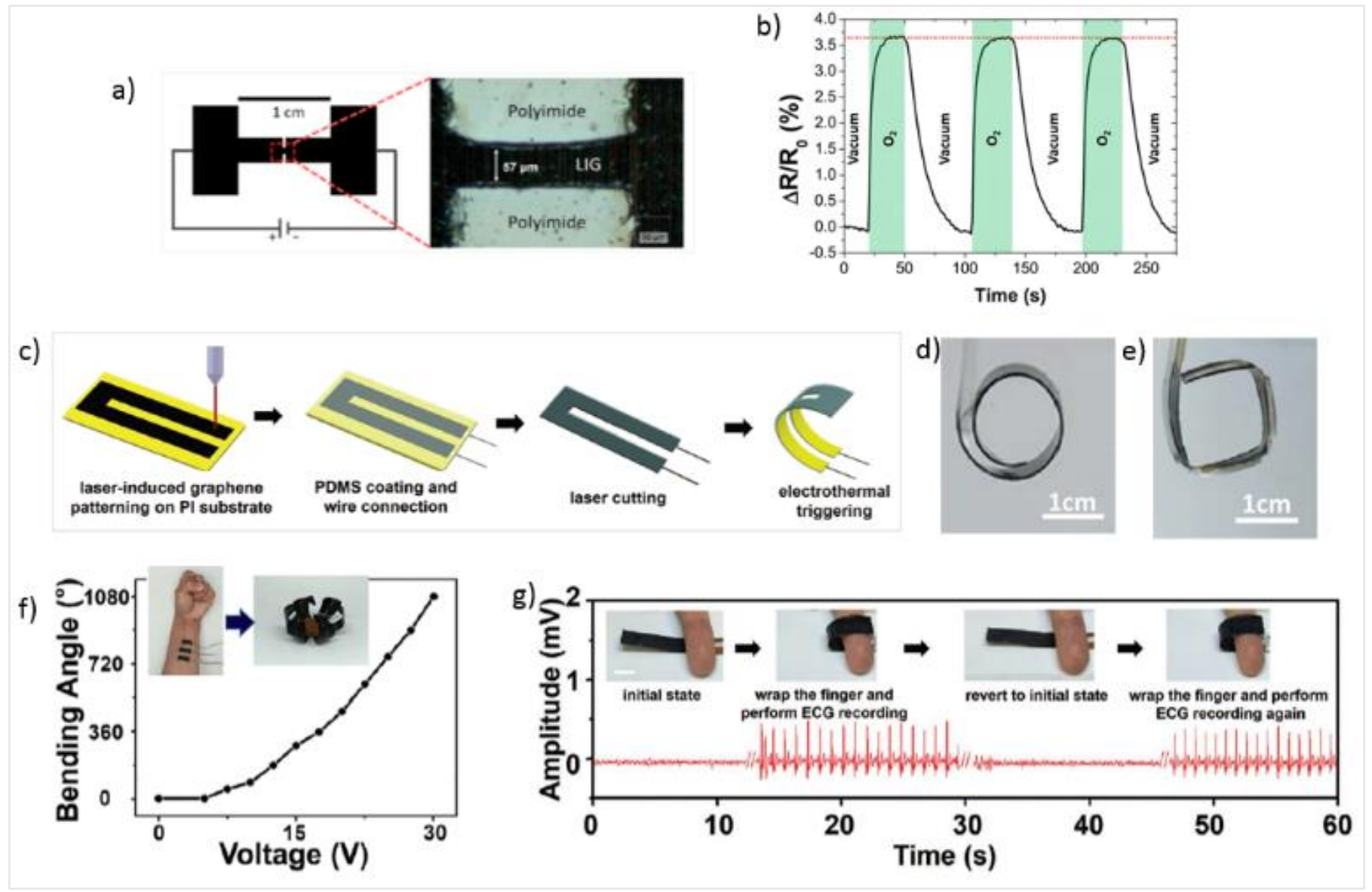

Fig. 7. a) Schematic of the LIG gas sensor with a zoomed-in active region [98]. b) The response of the LIG gas sensor to oxygen, at $12 \mathrm{~V}$ supply voltage [98] c) Fabrication process of a LIG electrothermal actuator[104]. d) Ring-like structure enabled by global bending [104]. e) Square-like structure enabled by local bending [104]. f) Bending angles as a function of the actuation voltage of the U-shaped LIG actuator [104]. g) Robotic finger to measure ECG signals in an on-demand and reversible fashion [104]. 
resulting in sound waves. The output sound pressure (SP) increased linearly with the input power, as shown in Fig. 8b. The artificial throat generated a wide-band sound with frequencies from $100 \mathrm{~Hz}$ to $40 \mathrm{kHz}$ [108].

The operation principle of LIG as a sound detector is based on the changes in the resistance of LIG upon weak vibrations. As illustrated in Fig. 8c, the resistance of LIG changed with human coughs, hums, and screams. The changes in resistance from different sounds were of different frequencies and magnitudes. However, the sound source developed by the above researchers is planar with obvious directivity. Whether the sound device can have an omnidirectional sound field when it is curved by an external force was not studied.

The directivity of the planar artificial throat [108] was recently improved by Zhang et al., who developed an omnidirectional sound device based on mechanically deformed LIG [96]. Its thermoacoustic performance was tested by applying $5 \mathrm{~V}$, generating Joule heat on the surface. The sound, produced through expansion and contraction of the air, was recorded by a microphone. The sound pressure had a linear relationship with the electrical input power and an inverse relationship with the distance from $0.5 \mathrm{~cm}$ to $5 \mathrm{~cm}$. The sound field of a planar device had a significant decrease at the backside of the PI. Meanwhile, the device wrapped around a cylinder (Fig. 8d), resulted in an omnidirectional sound field with no noticeable degradation after $60 \mathrm{~min}$. of operation (Fig.8e) [96].

\section{Magnetic-field transducers}

Recent discoveries have shown that LIG could be used for detecting and measuring the magnetic field via Magnetoresistance (MR) and Hall Effects. Both are common methods used in magnetic field sensors and will be discussed in this section. Benefiting from the versatility of the laser writing technology, LIG magnetic transducers could be easily integrated into ultra-thin navigation modules, body monitoring, and position monitoring systems.

Magnetoresistive sensors. The basic definition of MR is the change in resistance in response to the application of a magnetic field [137]. The investigation of the MR properties of porous LIG showed that it exhibits a nonsaturating negative MR response at temperatures ranging from $10 \mathrm{~K}$ to $300 \mathrm{~K}$. This finding was in contrast to conventional graphene with positive MR response at all temperatures [138].

A four-probe assembly was used to characterize the sensor. The distance between the middle two electrodes was $2 \mathrm{~mm}$, while the distance between the end electrodes was $6 \mathrm{~mm}$ (Fig. 9a). The MR was then calculated using

$$
\operatorname{MR}[\%]=\left(\frac{R_{B}}{R_{0}}-1\right) \cdot 100 \%,
$$

where $R_{B}$ is the resistance under the applied magnetic field, and $R_{0}$ is the resistance under zero magnetic field. The lowest MR was detected at $300 \mathrm{~K}$, while the highest MR magnitude was obtained at $10 \mathrm{~K}$ (Fig. 9b). The resistance as a function of temperature curve under zero magnetic field was used to determine the dominant conduction mechanism in the specimens. In this regard, the variable range hopping model was the most suitable due to the presence of defects in LIG and the temperature dependence of MR. The authors concluded that negative MR was driven by hopping conduction and quantum interference [106]. The negative $\mathrm{MR}$ was also tuned by annealing the LIG samples at $400^{\circ} \mathrm{C}$. Although the magnetotransport properties of the sensor were improved, the additional processing step involved a long exposure in a CVD furnace.
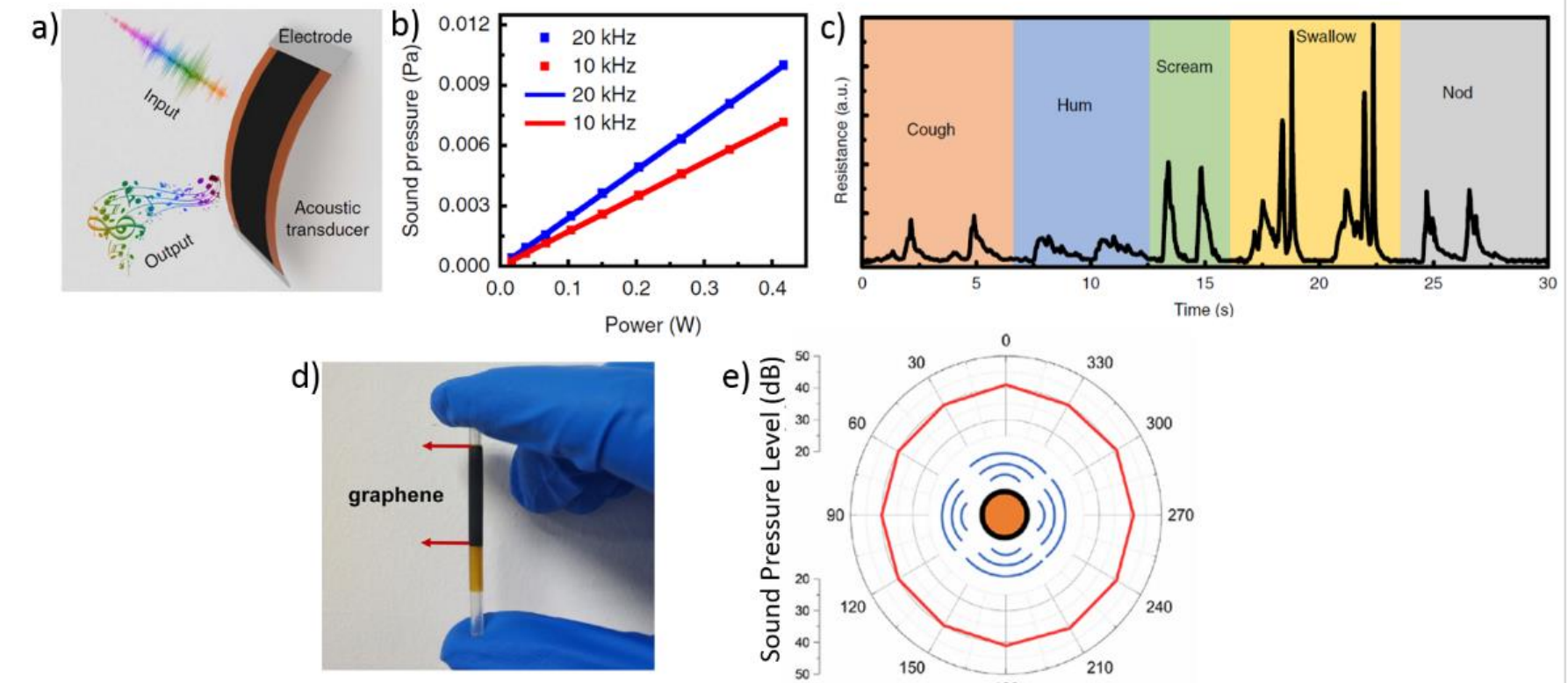

e)

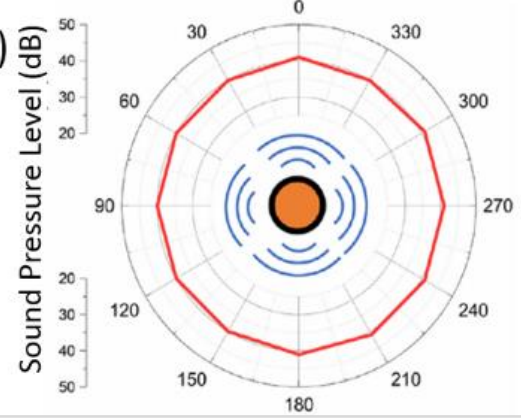

Fig. 8. a) LIG artificial throat with the ability to emitting and detecting sound [108]. b) Sound pressure versus input power at 10 and $20 \mathrm{kHz}$. The square and line represents the experimental and theoretical results, respectively [108]. c) Response of the LIG throat to coughs, hums, screams, swallowing, and nods of a human [108]. d) LIG attached to an ultrathin cylinder structure (radius of $1.5 \mathrm{~mm}$ ) [96] and e) the corresponding sound field [96]. 

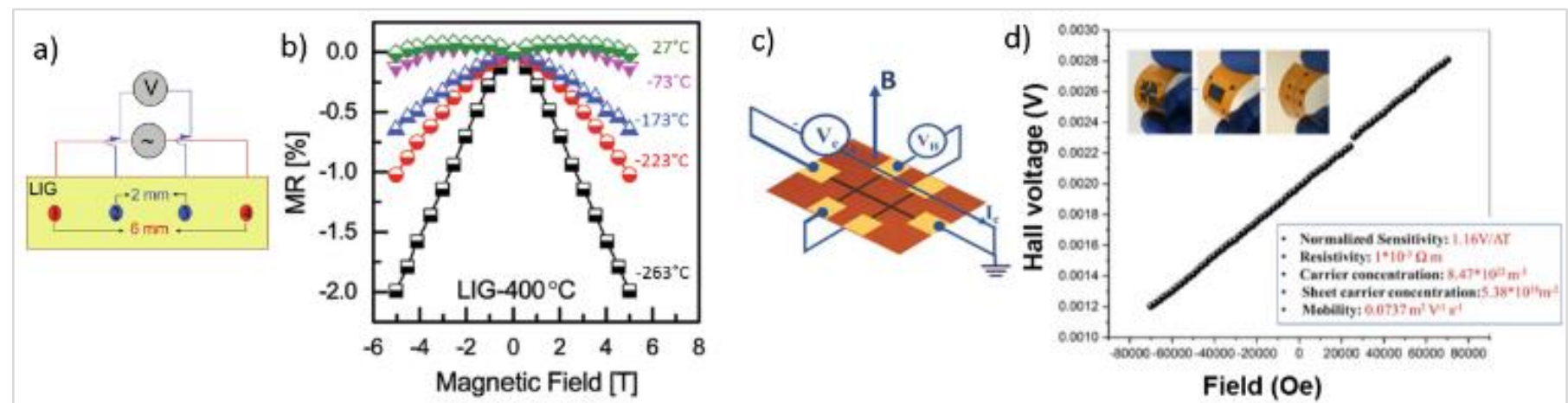

Fig. 9. a) Four-probe measurements of LIG magnetoresistance sensor [106]. b) MR of the LIG sensor [106]. C) LIG Hall-ettect sensor [91] d) its response to a magnetic field at $100 \mu \mathrm{A}$ bias current [91].

Hall-effect sensors. The Hall Effect in a device is characterized by the generation of a transverse electric field upon application of an electric current and a magnetic field perpendicular to the current. A LIG-based Hall Effect sensor was reported as part of a flexible and multifunctional graphene sensor platform [91]. The device architecture and geometry is shown in Fig 9c. The measured Hall voltage, $V_{H}$, in response to the transversely applied magnetic field, ranging from $-7 \mathrm{~T}$ to $7 \mathrm{~T}$, is shown in Fig. 9d, with a constant current flow of $100 \mu \mathrm{A}$.

The Hall voltage showed a linear dependence on the applied magnetic field with a current-normalized sensitivity of 1.16 V/AT, extracted from the slope of the response. Compared to CMOS Hall sensors, LIG Hall sensors have a higher carrier density $\left(\approx 10^{14} \mathrm{~cm}^{-2}\right)$ and lower carrier mobility $\left(\sim 737 \mathrm{~cm}^{2} \mathrm{~V}^{-}\right.$ ${ }^{1} \mathrm{~s}^{-1}$ ), both of which contributed to a lower Hall sensitivity. Nevertheless, the sensitivity of the sensor showed no degradation after being bent to a minimum radius of $5 \mathrm{~mm}$, and after being exposed to a corrosive seawater environment for an extended period of time [91].

\section{Electromagnetic transducers}

In this section, we discuss the interaction of electromagnetic waves with LIG. In particular, the exploitation of LIG in the microwave, radio wave $(\mathrm{RF})$ and terahertz $(\mathrm{THz})$ regimes and in solar-to-thermal conversion applications.

Solar tansducers. Sunlight, or the solar radiation spectrum, includes wavelengths between $240 \mathrm{~nm}$ and $2.5 \mu \mathrm{m}$, which encompasses ultraviolet, visible, and infrared radiation [139]. Li et al. have used LIG as a sunlight-absorbing material that converted the absorbed solar irradiation into heat and increased the temperature of water to produce steam. The heat was localized at the air-water interface [46], and the LIG evaporated the seawater into freshwater via solar energy with high efficiency and large scalability. A strong solar absorption, low thermal conductivity, and open porosity for capillarity contributed to the high-performance in solar-powered evaporation[46]. The top layer of LIG consisted of nano-scaled pores, while the bottom layer consisted of non-fully carbonized PI. This structure was termed as Janus membrane, since the static contact angle of water on the top side was almost $0^{\circ}$ (superhydrophilic), while the contact angle of the bottom side was over $150^{\circ}$ (superhydrophobic). In the test setup, the LIG film floated on the water surface of a beaker, as shown in Fig.10a. The weight losses of water as a result of one sun illumination is shown in Fig. 10b. The LIG obtained from 50 $\mu \mathrm{m}$ thick PI showed the highest rate of evaporation of up to 1.37 $\mathrm{kg} \mathrm{m}^{-2} \mathrm{~h}^{-1}$. A solar evaporation efficiency of $\sim 90 \%$ and an optical absorption rate of $99.9 \%$ were achieved, while the desalinated water possessed even higher purity than domestic water [46]. It should be noted that a very thin PI film $(\sim 50 \mu \mathrm{m})$ was utilized to achieve a sufficient capillary effect for pumping water; however, it compromised the thermal insulating properties of the membrane.

Microwave transducers. Microwave resonant sensors exploit the change in the spectral response of the resonance frequency a)
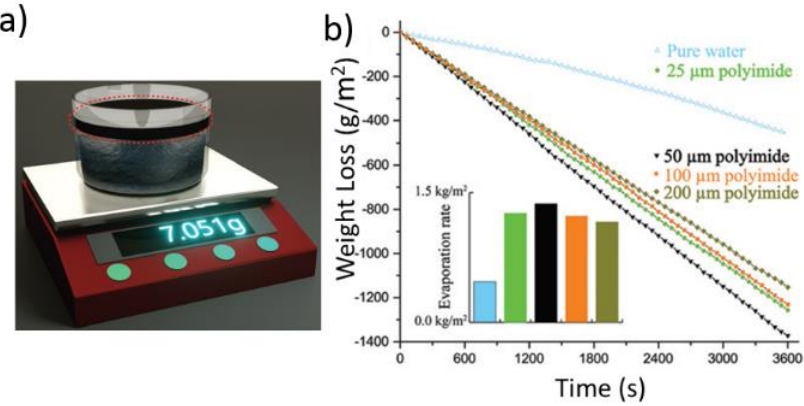

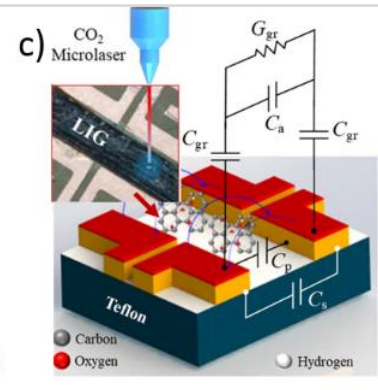

Fig. 10. a) The setup used to measure the weight loss of water [46]. b) The evaporation mass loss of water as a function of time [46]. The inset graph shows the evaporation rates for LIG obtained from different thicknesses of PI. c) Equivalent electric circuit of the coupled sensing region by incorporating humidified LIG [107]. d) Central frequency shifts as a function of humidity [107]. 
to realize sensitive measurements of a wide range of physical phenomena [140]. This process is linked to the change in a material's electromagnetic properties at $\mathrm{GHz}$ frequencies. Adhikari et al. exploited LIG in the microwave regime by integrating it into the capacitive sensing region of a planar microwave resonator [107]. The resonator was utilized to measure the relative humidity $(\mathrm{RH})$ of the environment, where porous LIG was used to increase the number of water molecule absorption sites in the sensing region. In particular, the patterned LIG was glued to the sensing region of a stepped impedance split-ring resonator, as shown in Fig. 10c. Fig. 10d shows the reflection coefficient $\left(S_{11}\right)$ under varying RH levels from which can be seen that the presence of a humid environment causes a downwards shift of the center frequency. The results suggested that this LIG microwave sensor responds linearly to a wide range of humidity levels (10\%-95\% RH) with high sensitivity $(70.93 \mathrm{kHz} / \% \mathrm{RH})$, low hysteresis $(0.375 \%$ $\mathrm{RH})$. The sensor, however, was limited by a long recovery time of at least $6 \mathrm{~s}$. As an energy source, microwave heating can provide internal and volumetric heating of materials. Along these lines, Xie et al. used LIG to absorb microwave energy and initiate synthesis of zeolitic imidazole frameworks [141]. It should be noted that LIG based humidity sensors were previously realized only in combination with graphene oxide (GO), which served as the humidity-sensitive material. These chemical sensors used oxygen contained groups to enhance the hydrophilicity and sensitivity to humidity [142-144].

Radio wave transducers. Inspired by the numerous reports on rapid heating of $\mathrm{sp}^{2}$-hybridized carbon nanostructures in response to electromagnetic radiation, Gerringer et al. investigated the heating ability of LIG in response to RF fields [93]. The method used RF fields to induce localized heating in contrast to uniform bulk heating from external sources such as ovens or furnaces [145]. LIG samples were exposed to RF fields with frequencies ranging from 50 to $145 \mathrm{MHz}$ at a constant power of $10 \mathrm{~W}$, which resulted in the rapid heating of
LIG with remarkable rates up to $126{ }^{\circ} \mathrm{C} / \mathrm{s}$. The temperature distribution across the LIG is shown in Fig.11a, where the hottest region $\left(220^{\circ} \mathrm{C}\right)$ was localized between the two copper strips with the strongest RF field. The heating response was largest in the frequency range of 100-120 MHz. However, it varied depending on the polymer utilized to produce LIG. The highest heating rate was achieved by LIG produced from polyethersulfone $\left(126^{\circ} \mathrm{C} / \mathrm{s}\right)$; meanwhile the one of $\mathrm{LIG}$ obtained from Kapton was only $79.55{ }^{\circ} \mathrm{C} / \mathrm{s}$. In addition, RF heaters were proposed for the rapid welding of polymer interfaces, such as PI, PEI, PEEK, and PC (listed in Fig. 1a). A solid weld was achieved in just 30s of RF exposure. Indeed, localized heat generated during RF field exposure of LIG promoted polymer diffusion and created a weld between two polymer surfaces [93] (Fig. 11b).

Terahertz wave transducers. $\mathrm{THz}$ radiation, which refers to electromagnetic waves with a frequency in the range of 0.1 to $10 \mathrm{THz}$, has been intensively investigated because of its great potential in the fields of information communication, medical sciences, and security [146]. Wang et al. fabricated a LIG-based THz grating and Fresnel zone plates (FZPs) [109].

Polarization-sensitive effects of the grating and focusing effects of the zone plates were observed via the $\mathrm{THz}$ focal plane imaging system, which detected the intensity and phase of the THz waves (Fig. 11c). The transmission spectra of LIG showed that the transmission was below 0.02 in the frequency range of 0.1-1 THz (Fig. 11d). It was concluded that LIG had the ability to block $\mathrm{THz}$ wave propagation due to its high conductivity [109]. The transmission spectra of the linearly polarized $\mathrm{THz}$ waves through the LIG grating were also measured. Fig. 11e shows the dependence of the transmission on the polarization. The polarization in the parallel direction had higher transmission than that in the vertical direction in the range from 0.1 to $0.5 \mathrm{THz}$, showing that the LIG grating had a polarizationselection effect for THz waves [146]. It should be noted that the polarization-selection effect was demonstrated for a limited
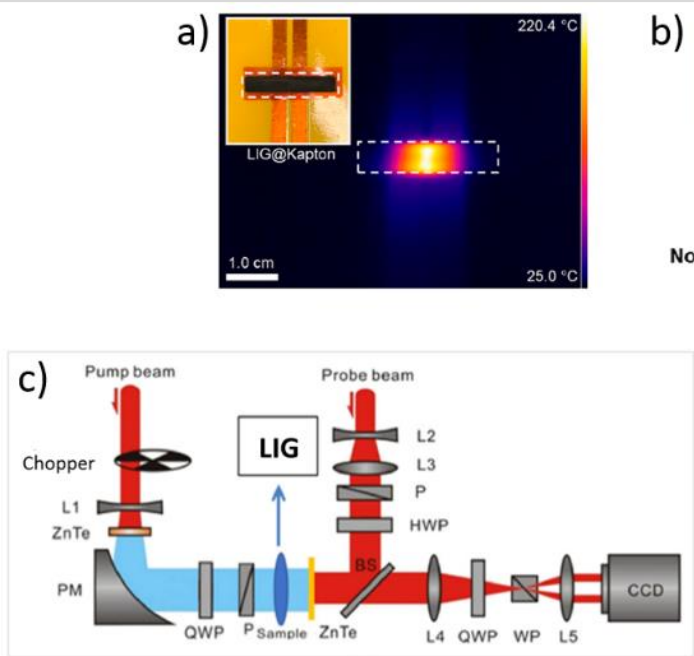

b)

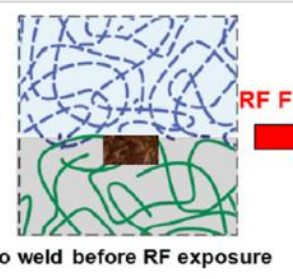

No weld before RF exposure

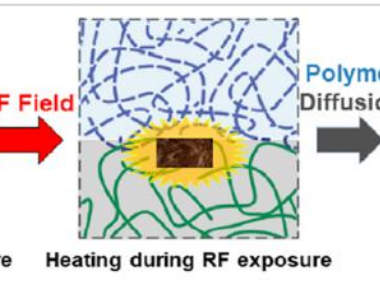

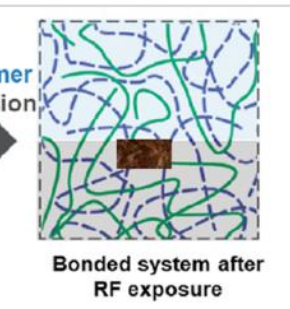

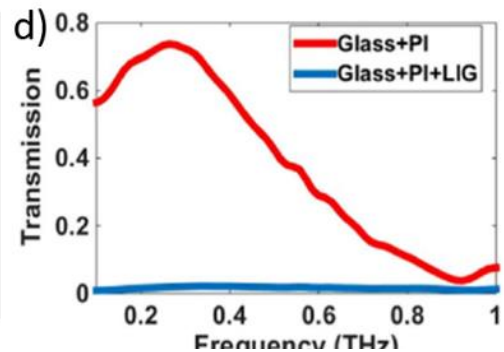

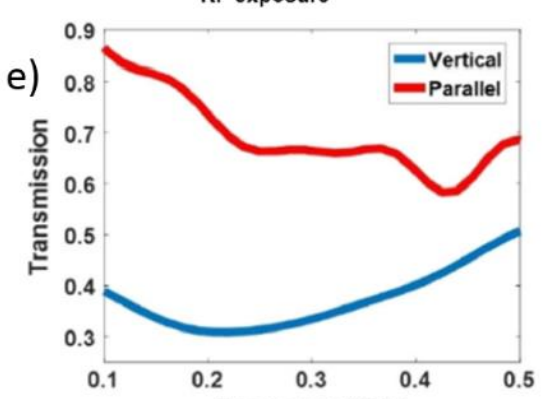

Fig. 11. a) Temperature distribution across LIG during RF field exposure [93]. b) LIG (black rectangle) heating upon exposure to RF fields, allowing for polymer chains to diffuse across the interface and form a weld [93]. c) Schematic of the THz focal plane imaging system[109]. d) Transmission spectra of the bare PI and LIG/PI on the glass substrate [109]. e) Transmission spectra of the THz waves parallel with and perpendicular to the LIG grating [109]. 
frequency range and not realized for higher frequency $\mathrm{THz}$ waves $(>0.5 \mathrm{~Hz})$. The authors anticipated that LIG might open up an avenue to achieve $\mathrm{THz}$ modulators that are low cost and easily fabricated.

\section{CONCLUSION AND OUTLOOK}

With the fast development of wearable consumer electronics, soft robotics, healthcare, and environmental monitoring, there is a strong need for high performance, flexible sensing platforms. To this end, physical sensors need to be as lightweight and compact as possible, often cover a large area, yet be able to survive the increasingly harsh environmental conditions[147], and, of course, their fabrication must be costeffective. The limitations of silicon-based micronanoelectronics have led researchers to identify new material platforms that can operate and survive within a high pressure, high temperature, and chemically corrosive environments for an extended period of time. Recent developments relying on advanced materials ( $\mathrm{SiC}, \mathrm{GaN}, \mathrm{AlN}$ ) and fabrication technologies, have low availability and high processing costs, which severely hinder their utilization.

LIG has emerged as a versatile and multifunctional material displaying physical coupling in several domains. This review has shown its suitability to be utilized for a mechanical, thermal, magnetic, and electromagnetic transducer functional material. It also provided insights into various design strategies established for preparing reliable physical sensors without the involvement of chemical treatments, synthesis, and multi-step fabrication processes. The effects of laser choice, lasing environment, and parameters on the graphene properties have been summarized. We also show a wide spectrum of applications of the LIG physical sensors in four different fields:

Table IV. Challenges and potential solutions to enable further development of LIG physical sensors,

\begin{tabular}{|c|c|c|}
\hline & Challenges & Potential solutions \\
\hline \multirow[t]{2}{*}{ Fabrication } & Slow printing process & $\begin{array}{l}\text { Parallel processing strategies, including the adoption of spatial light modulators, holographic laser } \\
\text { processing, multilayer beams and/or patterned light field to be developed. A major leap forward } \\
\text { can be expected by implementing roll-to-roll fabrication to increase the yield, like in many printing } \\
\text { technologies. }\end{array}$ \\
\hline & Limited resolution & $\begin{array}{l}\text { Alternative lasers with smaller focal spot sizes are needed to achieve high spatial resolution. The } \\
\text { possibilities of femtosecond - laser direct writing and vertical LIG architectures are attractive } \\
\text { options for future investigations. }\end{array}$ \\
\hline \multirow[t]{2}{*}{ Performance } & Cross-sensitivity & $\begin{array}{l}\text { Effective decoupling algorithms should be realized to avoid interference between the multiple } \\
\text { stimuli for specific applications. }\end{array}$ \\
\hline & Motion induced noise & $\begin{array}{l}\text { Mechanical noise could be reduced by using a redundant sensor, while applying algorithms to pick } \\
\text { up the real signals from noise. }\end{array}$ \\
\hline \multirow{2}{*}{ Applications } & $\begin{array}{l}\text { Sensor-human } \\
\text { interface }\end{array}$ & $\begin{array}{l}\text { Benefitting from low cytotoxicity and biocompatibility of LIG, LIG physical sensors could be tested } \\
\text { on patients as part of clinical trials. The comfortability and compliance of sensors need to be } \\
\text { systematically assessed. }\end{array}$ \\
\hline & $\begin{array}{l}\text { Internet of things } \\
\text { (smart home, city, } \\
\text { grids, etc.) }\end{array}$ & $\begin{array}{l}\text { Effective loT connectivity protocols should be selected to provide a trade-off between power and } \\
\text { bandwidth consumption. The reliability of LIG physical sensors could be then evaluated in } \\
\text { crowded, noisy and more complex environments. }\end{array}$ \\
\hline
\end{tabular}

healthcare, environmental monitoring, robotics, and quality control.

The scope of its applications has been broadened by its advantageous features, such as low cytotoxicity [90], biocompatibility [148, 149], corrosion resistance [92] and ability to operate in high temperatures $\left(>400^{\circ} \mathrm{C}\right)[102]$. The properties of LIG can be easily tailored during a single-step laser irradiation process by a variety of lasers, while its morphology, composition, and surface area can be tuned via lasing parameters and the surrounding environment. The sensitivities and dynamic ranges of transducers can be customized by their geometries, sizes, and shapes.

It should be mentioned that preparing graphene-based materials by ink-jet, screen printing, and roll coating techniques often involve the use of solvents and additional binders. Though these techniques offer reliable patterning of graphene materials, the performance is often limited, due to the agglomeration of graphene layers. Meanwhile, laser-based technologies proved to be innovative and scalable approaches for direct-write fabrication of 3D graphene electrodes.

Although many LIG based physical sensors have proven their feasibility and reliability, there are several challenges that need to be tackled. The summary of these challenges with some potential solution paths are provided in Table IV. For the LIG technology to target the mass market, it has to show a route for high-throughput and low-cost production. In particular, the laser scan rate should be ramped up without sacrificing the LIG properties, whilst maintaining a good part-to-part tolerance. As seen in Fig. 5, to obtain 3D porous graphene, $\mathrm{CO}_{2}$ lasers use speeds in the range of $\sim 2$ to $6 \mathrm{~m} / \mathrm{min}$. Although this laser provides a faster route to prepare graphene than conventional CVD and exfoliation processes, the scribing rate is insufficient to compete with the production of some alternative conductive materials, such as copper, aluminum, and silver. These 
materials can be currently roll-to-roll stamped out on a machine that runs at up to $30 \mathrm{~m} / \mathrm{min}$, producing 250,000 units per hour[150]. Conductive metals can also be printed using flex printers running at $40 \mathrm{~m} / \mathrm{min}$, although here, curing times slow down the yield. For instance, the main competitor for the LIG strain sensor is the incumbent metal foil strain gauge technology. The primary challenge facing the commercialization of LIG strain sensors is that the incumbent is a simple, well- established, and low-cost technology.

However, the LIG method is amenable for roll-to-roll fabrication, creating opportunities for scaled-up production in the future. Another challenge faced by direct lasing technology is the need to increase the packing density or number of devices printed per unit area. The smallest reported spatial resolution of LIG $(\sim 12 \mu \mathrm{m})$ is on a micro-scale and comparable with many methods of printed electronics; however, it is inadequate for next-generation, miniaturized, and compact sensors.

Therefore, future research efforts need to be directed towards obtaining a reduced line width at the nanometer scale. Fabrication of vertical graphene architectures should also be investigated to increase the LIG density in a given footprint area for improved areal performance metrics.

Since LIG could respond to multiple physical stimuli simultaneously, it could be challenging to separate the type and the intensity of each stimulus. This means that selective sensing devices with low cross-sensitivity and effective decoupling algorithms are needed to avoid interference between multiple stimuli for specific end applications. The integration of LIG transducers with other flexible electronics could also be challenging, due to the mismatch in mechanical, thermal, and electrical characteristics. Multilevel interconnections such as stretchable conductors would be required to preserve structural integrity while delivering power and data in a stable manner. The investigation of information processing interfaced with LIG sensors is an appealing research area that may lead to many frontier applications, such as artificial intelligence, intelligent robotics, medical monitoring, and healthcare measurement.

\section{ACKNOWLEDGMENT}

This research was funded by King Abdullah University of Science and Technology (KAUST).

\section{REFERENCES}

1. Yeo, J.C. and C.T. Lim, Emerging flexible and wearable physical sensing platforms for healthcare and biomedical applications. Microsystems \& Nanoengineering, 2016. 2(1): p. 1-19.

2. $\quad$ !!! INVALID CITATION !!! [2-4].

3. Chang, T.-H., et al., Stretchable graphene pressure sensors with Shar-Pei-like hierarchical wrinkles for collision-aware surgical robotics. ACS applied materials \& interfaces, 2019. 11(10): p. 10226-10236.

4. Seminara, L., et al., Electromechanical characterization of piezoelectric PVDF polymer films for tactile sensors in robotics applications. Sensors and Actuators A: Physical, 2011. 169(1): p. 49-58.

5. Florez, J. and C. Parra. Review of sensors used in robotics for humanitarian demining application. in 2016 IEEE Colombian Conference on Robotics and Automation (CCRA). 2016. IEEE.

6. Takei, K., et al., Physical and chemical sensing with electronic skin. Proceedings of the IEEE, 2019. 107(10): p. 2155-2167.
Lou, Z., et al., An ultra-sensitive and rapid response speed graphene pressure sensors for electronic skin and health monitoring. Nano Energy, 2016. 23: p. 7-14.

8. Zhao, S. and R. Zhu, Electronic skin with multifunction sensors based on thermosensation. Advanced Materials, 2017. 29(15): p. 1606151.

9. Bergmann, J.H., et al., An attachable clothing sensor system for measuring knee joint angles. IEEE Sensors Journal, 2013. 13(10): p. 4090-4097.

10. Wang, F., et al., Flexible pressure sensors for smart protective clothing against impact loading. Smart materials and structures, 2013. 23(1): p. 015001.

11. Nanjappan, V., et al. Clothing-based wearable sensors for unobtrusive interactions with mobile devices. in 2017 International SoC Design Conference (ISOCC). 2017. IEEE.

12. Islam, T., S.C. Mukhopadhyay, and N.K. Suryadevara, Smart sensors and internet of things: A postgraduate paper. IEEE Sensors Journal, 2016. 17(3): p. 577-584.

13. Madakam, S., et al., Internet of Things (IoT): A literature review. Journal of Computer and Communications, 2015. 3(05): p. 164.

14. Zhu, C., et al., Green internet of things for smart world. IEEE access, 2015. 3: p. 2151-2162.

15. Bai, Y., et al., Flexible and Wearable Electronics: from Lab to Fab. Flexible and Wearable Electronics for Smart Clothing, 2020: p. 285303.

16. Trung, T.Q. and N.E. Lee, Flexible and stretchable physical sensor integrated platforms for wearable human-activity monitoringand personal healthcare. Advanced materials, 2016. 28(22): p. 43384372.

17. Ge, G., et al., Recent progress of flexible and wearable strain sensors for human-motion monitoring. Journal of Semiconductors, 2018. 39(1): p. 011012.

18. Zheng, Q., et al., Graphene-based wearable piezoresistive physical sensors. Materials Today, 2020.

19. Yu, X., et al., Graphene-based smart materials. Nature Reviews Materials, 2017. 2(9): p. 1-13.

20. Geim, A.K. and K.S. Novoselov, The rise of graphene, in Nanoscience and technology: a collection of reviews from nature journals. 2010, World Scientific. p. 11-19.

21. Kostarelos, K. and K.S. Novoselov, Graphene devices for life. Nature nanotechnology, 2014. 9(10): p. 744-745.

22. Geim, A.K., Graphene: status and prospects. science, 2009. 324(5934): p. 1530-1534.

23. Blake, P., et al., Making graphene visible. Applied physics letters, 2007. 91(6): p. 063124.

24. Fang, Q., Y. Shen, and B. Chen, Synthesis, decoration and properties of three-dimensional graphene-based macrostructures: $a$ review. Chemical Engineering Journal, 2015. 264: p. 753-771. Kuang, J., et al., A hierarchically structured graphene foam and its potential as a large-scale strain-gauge sensor. Nanoscale, 2013. 5(24): p. 12171-12177.

26. Kotal, M., et al., Recent progress in multifunctional graphene aerogels. Frontiers in Materials, 2016. 3: p. 29.

27. Ismach, A., et al., Direct chemical vapor deposition of graphene on dielectric surfaces. Nano letters, 2010. 10(5): p. 1542-1548.

28. Sun, J., et al., Low partial pressure chemical vapor deposition of graphene on copper. IEEE transactions on nanotechnology, 2011. 11(2): p. 255-260.

29. Yan, Z., Z. Peng, and J.M. Tour, Chemical vapor deposition of graphene single crystals. Accounts of chemical research, 2014. 47(4): p. 1327-1337.

30. He, R., et al., Large physisorption strain in chemical vapor deposition of graphene on copper substrates. Nano letters, 2012. 12(5): p. 2408-2413.

31. Plutnar, J., M. Pumera, and Z. Sofer, The chemistry of CVD graphene. Journal of Materials Chemistry C, 2018. 6(23): p. 60826101.

32. Zhang, Y., et al., Comparison of graphene growth on singlecrystalline and polycrystalline Ni by chemical vapor deposition. The Journal of Physical Chemistry Letters, 2010. 1(20): p. 3101-3107.

33. Martins, L.G., et al., Direct transfer of graphene onto flexible substrates. Proceedings of the National Academy of Sciences, 2013. 110(44): p. 17762-17767. 
Yoon, T., et al., Healing graphene defects using selective electrochemical deposition: toward flexible and stretchable devices. ACS nano, 2016. 10(1): p. 1539-1545.

35. Leong, W.S., et al., Paraffin-enabled graphene transfer. Nature communications, 2019. 10(1): p. 1-8

36. Hill, E.W., A. Vijayaragahvan, and K. Novoselov, Graphene sensors. IEEE Sensors Journal, 2011. 11(12): p. 3161-3170.

37. Ciesielski, A. and P. Samori, Graphene via sonication assisted liquid-phase exfoliation. Chemical Society Reviews, 2014. 43(1): p. 381-398.

38. Cui, X., et al., Liquid-phase exfoliation, functionalization and applications of graphene. Nanoscale, 2011. 3(5): p. 2118-2126.

39. Ciesielski, A. and P. Samori, Supramolecular Approaches to Graphene: From Self-Assembly to Molecule-Assisted Liquid-Phase Exfoliation. Advanced materials, 2016. 28(29): p. 6030-6051.

Lin, J., et al., Laser-induced porous graphene films from commercial polymers. Nature communications, 2014. 5(1): p. 1-8.

41. Vashisth, A., et al., ReaxFF Simulations of Laser-Induced Graphene (LIG) Formation for Multifunctional Polymer Nanocomposites. ACS Applied Nano Materials, 2020. 3(2): p. 1881-1890.

42. Ye, R., D.K. James, and J.M. Tour, Laser-induced graphene. Accounts of chemical research, 2018. 51(7): p. 1609-1620.

43. Sha, Y., et al., Laser induced graphitization of PAN-based carbon fibers. RSC advances, 2018. 8(21): p. 11543-11550.

44. Carvalho, A.F., et al., Laser-induced graphene strain sensors produced by ultraviolet irradiation of polyimide. Advanced Functional Materials, 2018. 28(52): p. 1805271.

45. Zhang, Z., et al., Visible light laser-induced graphene from phenolic resin: A new approach for directly writing graphene-based electrochemical devices on various substrates. Carbon, 2018. 127: p. 287-296.

46. Li, G., W.-C. Law, and K.C. Chan, Floating, highly efficient, and scalable graphene membranes for seawater desalination using solar energy. Green Chemistry, 2018. 20(16): p. 3689-3695.

47. Li, Y., et al., Laser-Induced Graphene in Controlled Atmospheres: From Superhydrophilic to Superhydrophobic Surfaces. Advanced Materials, 2017. 29(27): p. 1700496.

48. Cai, J., C. Lv, and A. Watanabe, Laser direct writing of highperformance flexible all-solid-state carbon micro-supercapacitors for an on-chip self-powered photodetection system. Nano Energy, 2016. 30: p. 790-800.

49. Nasser, J., et al., Laser induced graphene fibers for multifunctional aramid fiber reinforced composite. Carbon, 2020. 158: p. 146-156.

50. Lamberti, A., et al., New insights on laser-induced graphene electrodes for flexible supercapacitors: tunable morphology and physical properties. Nanotechnology, 2017. 28(17): p. 174002.

51. Duy, L.X., et al., Laser-induced graphene fibers. Carbon, 2018. 126: p. 472-479.

52. Cardoso, A.R., et al., Molecularly-imprinted chloramphenicol sensor with laser-induced graphene electrodes. Biosensors and Bioelectronics, 2019. 124: p. 167-175.

53. Beduk, T., et al., One-step electrosynthesized molecularly imprinted polymer on laser scribed graphene bisphenol a sensor. Sensors and Actuators B: Chemical, 2020: p. 128026.

54. Wang, F., et al., Laser-induced graphene: preparation, functionalization and applications. Materials technology, 2018. 33(5): p. 340-356.

55. Zhang, Y., et al., A flexible non-enzymatic glucose sensor based on copper nanoparticles anchored on laser-induced graphene. Carbon, 2020. 156: p. 506-513.

56. Yang, Y., et al., A laser-engraved wearable sensor for sensitive detection of uric acid and tyrosine in sweat. Nature Biotechnology, 2020. 38(2): p. 217-224.

57. Hui, X., et al., A highly flexible and selective dopamine sensor based on Pt-Au nanoparticle-modified laser-induced graphene. Electrochimica Acta, 2019. 328: p. 135066.

58. Fenzl, C., et al., Laser-scribed graphene electrodes for aptamerbased biosensing. ACS sensors, 2017. 2(5): p. 616-620.

59. Ye, R., D.K. James, and J.M. Tour, Laser-induced graphene: from discovery to translation. Advanced Materials, 2019. 31(1): p. 1803621 .

60. Li, G., Direct laser writing of graphene electrodes. Journal of Applied Physics, 2020. 127(1): p. 010901.
61. Kurra, N., et al., Laser-derived graphene: a three-dimensional printed graphene electrode and its emerging applications. Nano Today, 2019. 24: p. 81-102.

62. Thamaraiselvan, C., et al., Laser-induced graphene and carbon nanotubes as conductive carbon-based materials in environmental technology. Materials Today, 2019.

63. Li, L., et al., High-performance pseudocapacitive microsupercapacitors from laser-induced graphene. Advanced Materials, 2016. 28(5): p. 838-845.

64. Ren, M., J. Zhang, and J.M. Tour, Laser-induced graphene synthesis of Co3O4 in graphene for oxygen electrocatalysis and metal-air batteries. Carbon, 2018. 139: p. 880-887.

65. Yoon, H., et al., A chemically modified laser-induced porous graphene based flexible and ultrasensitive electrochemical biosensor for sweat glucose detection. Sensors and Actuators B: Chemical, 2020. 311: p. 127866.

66. Cai, J., et al., Laser direct writing of heteroatom-doped porous carbon for high-performance micro-supercapacitors. Energy Storage Materials, 2020. 25: p. 404-415.

67. Wang, F., et al., Laser-induced nitrogen-doped hierarchically porous graphene for advanced electrochemical energy storage. Carbon, 2019. 150: p. 396-407.

68. Basu, A., et al., CO2 laser direct written MOF-based metaldecorated and heteroatom-doped porous graphene for flexible allsolid-state microsupercapacitor with extremely high cycling stability. ACS Applied Materials \& Interfaces, 2016. 8(46): p. 31841-31848.

69. Zhang, C., et al., Monolithic and flexible ZnS/SnO2 ultraviolet photodetectors with lateral graphene electrodes. Small, 2017. 13(18): p. 1604197.

70. Ge, L., et al., A laser-induced TiO 2-decorated graphene photoelectrode for sensitive photoelectrochemical biosensing. Chemical Communications, 2019. 55(34): p. 4945-4948.

71. Yagati, A.K., et al., Laser-induced graphene interdigitated electrodes for label-free or nanolabel-enhanced highly sensitive capacitive aptamer-based biosensors. Biosensors and Bioelectronics, 2020: p. 112272.

72. Zhu, J., et al., Biomimetic turbinate-like artificial nose for hydrogen detection based on $3 D$ porous laser-induced graphene. ACS applied materials \& interfaces, 2019. 11(27): p. 24386-24394.

73. You, R., et al., Laser fabrication of graphene-based flexible electronics. Advanced Materials, 2020. 32(15): p. 1901981.

74. Liu, Y.-Q., et al., Laser fabrication of graphene-based electronic skin. Frontiers in chemistry, 2019. 7: p. 461.

75. Kumar, R., et al., Laser-assisted synthesis, reduction and micropatterning of graphene: recent progress and applications. Coordination Chemistry Reviews, 2017. 342: p. 34-79.

76. Wan, Z., et al., Laser-Reduced Graphene: Synthesis, Properties, and Applications. Advanced Materials Technologies, 2018. 3(4): p. 1700315.

77. Biswas, R.K., et al., Improved conductivity of carbonized polyimide by $\mathrm{CO} 2$ laser graphitization. Journal of Materials Chemistry C, 2020. 8(13): p. 4493-4501.

78. Huang, Y., et al., Laser Direct Writing of Heteroatom ( $N$ and $S)$ Doped Graphene from a Polybenzimidazole Ink Donor on Polyethylene Terephthalate Polymer and Glass Substrates. Small, 2018. 14(44): p. 1803143.

79. Zhu, C., et al., Direct laser writing of graphene films from a polyether ether ketone precursor. Journal of Materials Science, 2019. 54(5): p. 4192-4201.

80. Lamberti, A., et al., A highly stretchable supercapacitor using laserinduced graphene electrodes onto elastomeric substrate. Advanced Energy Materials, 2016. 6(10): p. 1600050.

81. Yazdi, A.Z., et al., Direct Creation of Highly Conductive LaserInduced Graphene Nanocomposites from Polymer Blends. Macromolecular Rapid Communications, 2017. 38(17): p. 1700176.

82. Wahab, H., et al., Machine-learning-assisted fabrication: Bayesian optimization of laser-induced graphene patterning using in-situ Raman analysis. Carbon, 2020.

83. Ferrari, A.C., et al., Raman spectrum of graphene and graphene layers. Physical review letters, 2006. 97(18): p. 187401.

84. Dong, H., S. Guo, and L. Zhao, Facile Preparation of Multilayered Graphene with CO2 as a Carbon Source. Applied Sciences, 2019. 9(21): p. 4482. 
Tittle, C.M., et al., Robust Superhydrophobic Laser-Induced Graphene for Desalination Applications. Advanced Materials Technologies, 2018. 3(2): p. 1700207.

86. Luo, S., P.T. Hoang, and T. Liu, Direct laser writing for creating porous graphitic structures and their use for flexible and highly sensitive sensor and sensor arrays. Carbon, 2016. 96: p. 522-531.

87. Kaidarova, A., et al., Flexible and biofouling independent salinity sensor. Advanced Materials Interfaces, 2018. 5(23): p. 1801110.

88. Kaidarova, A., et al., Flexible conductivity, temperature, and depth sensor for marine environment monitoring. 2019.

89. Kaidarova, A., et al., Wearable multifunctional printed graphene sensors. npj Flexible Electronics, 2019. 3(1): p. 1-10.

90. Kaidarova, A., et al., Laser-Printed, Flexible Graphene Pressure Sensors. Global Challenges, 2020: p. 2000001.

91. Kaidarova, A., et al. Flexible and Multi-functional Graphene sensor platform. in 2019 IEEE International Conference on Flexible and Printable Sensors and Systems (FLEPS). 2019. IEEE.

92. Khan, M.A., et al., Magnetic Composite Hydrodynamic Pump With Laser-Induced Graphene Electrodes. IEEE Transactions on Magnetics, 2017. 53(11): p. 1-4.

93. Gerringer, J.C., et al., Radio Frequency Heating of Laser-Induced Graphene on Polymer Surfaces for Rapid Welding. ACS Applied Nano Materials, 2019. 2(11): p. 7032-7042.

94. Han, T., et al., Multifunctional flexible sensor based on laserinduced graphene. Sensors, 2019. 19(16): p. 3477.

95. Zhu, J., et al., Cost-effective fabrication and high-frequency response of non-ideal $R C$ application based on $3 D$ porous laserinduced graphene. Journal of Materials Science, 2018. 53(17): p. 12413-12420.

96. Zhang, P., et al., Flexible laser-induced-graphene omnidirectional sound device. Chemical Physics Letters, 2020: p. 137275.

97. $\quad \mathrm{Wu}, \mathrm{Y}$. ., et al., Piezoresistive stretchable strain sensors with human machine interface demonstrations. Sensors and Actuators A: Physical, 2018. 279: p. 46-52.

98. Stanford, M.G., et al., Laser-induced graphene for flexible and embeddable gas sensors. ACS nano, 2019. 13(3): p. 3474-3482.

99. Rahimi, R., et al., Highly stretchable and sensitive unidirectional strain sensor via laser carbonization. ACS applied materials \& interfaces, 2015. 7(8): p. 4463-4470.

100. Stanford, M.G., et al., Laser-induced graphene triboelectric nanogenerators. ACS nano, 2019. 13(6): p. 7166-7174.

101. Luong, D.X., et al., Laminated object manufacturing of 3D-printed laser-induced graphene foams. Advanced Materials, 2018. 30(28): p. 1707416.

102. Stanford, M.G., et al., Self-sterilizing laser-induced graphene bacterial air filter. ACS nano, 2019. 13(10): p. 11912-11920.

103. Tian, Q., et al., Bean Pod-Inspired Ultrasensitive and Self-Healing Pressure Sensor Based on Laser-Induced Graphene and Polystyrene Microsphere Sandwiched Structure. ACS applied materials \& interfaces, 2020. 12(8): p. 9710-9717.

104. Ling, Y., et al., Laser-Induced Graphene for Electrothermally Controlled, Mechanically Guided, 3D Assembly and Human-Soft Actuators Interaction. Advanced Materials, 2020. 32(17): p. 1908475.

105. Dallinger, A., et al., Stretchable and skin-conformable conductors based on Polyurethane/Laser-Induced Graphene. ACS Applied Materials \& Interfaces, 2020. 12(17): p. 19855-19865.

106. Li, X., et al., Nonsaturating negative magnetoresistance in laserinduced graphene. Materials Letters, 2019. 248: p. 43-47.

107. Adhikari, K.K., et al., Polyimide-derived laser-induced porous graphene-incorporated microwave resonator for high-performance humidity sensing. Applied Physics Express, 2019. 12(10): p. 106501.

108. Tao, L.-Q., et al., An intelligent artificial throat with sound-sensing ability based on laser induced graphene. Nature communications, 2017. 8(1): p. 1-8.

109. Wang, Z., et al., Patterned laser-induced graphene for terahertz wave modulation. JOSA B, 2020. 37(2): p. 546-551.

110. Bobinger, M.R., et al., Flexible and robust laser-induced graphene heaters photothermally scribed on bare polyimide substrates. Carbon, 2019. 144: p. 116-126.

111. Stanford, M.G., et al., High-Resolution Laser-Induced Graphene. Flexible Electronics beyond the Visible Limit. ACS Applied Materials \& Interfaces, 2020. 12(9): p. 10902-10907.
112. Chen, Y., et al., UV Laser-Induced Polyimide-to-Graphene Conversion: Modeling, Fabrication, and Application. Small Methods, 2019. 3(10): p. 1900208.

113. Singh, E., et al., Superhydrophobic graphene foams. small, 2013. 9(1): p. 75-80

114. Ye, R., et al., Laser-induced graphene formation on wood. Advanced Materials, 2017. 29(37): p. 1702211.

115. Peng, Z., et al., Flexible and stackable laser-induced graphene supercapacitors. ACS applied materials \& interfaces, 2015. 7(5): p. 3414-3419.

116. Kumar, P., K. Subrahmanyam, and C. Rao, Graphene produced by radiation-induced reduction of graphene oxide. International Journal of Nanoscience, 2011. 10(04n05): p. 559-566.

117. Chen, X., et al., A dual-functional graphene-based self-alarm health-monitoring E-skin. Advanced Functional Materials, 2019. 29(51): p. 1904706.

118. Marengo, M., G. Marinaro, and J. Kosel. Flexible temperature and flow sensor from laser-induced graphene. in 2017 IEEE SENSORS. 2017. IEEE

119. Nasser, J., et al., Laser induced graphene printing of spatially controlled super-hydrophobic/hydrophilic surfaces. Carbon, 2020.

120. Luong, D.X., Laser Induced Graphene Nanomaterials and Applications. 2017.

121. Dong, J., et al., Control of superhydrophilic and superhydrophobic graphene interface. Scientific reports, 2013. 3: p. 1733.

122. Pang, Y., et al., Multifunctional mechanical sensors for versatile physiological signal detection. ACS applied materials \& interfaces, 2018. 10(50): p. 44173-44182.

123. Yu, D.Y. and F. Spaepen, The yield strength of thin copper films on Kapton. Journal of Applied Physics, 2004. 95(6): p. 2991-2997.

124. Cao, H., et al., Development and characterization of a novel interdigitated capacitive strain sensor for structural health monitoring. IEEE Sensors Journal, 2015. 15(11): p. 6542-6548.

125. Zhao, P., et al., Replacing the metal electrodes in triboelectric nanogenerators: High-performance laser-induced graphene electrodes. Nano Energy, 2020: p. 104958.

126. Rahimi, R., M. Ochoa, and B. Ziaie, Direct laser writing of porouscarbon/silver nanocomposite for flexible electronics. ACS applied materials \& interfaces, 2016. 8(26): p. 16907-16913.

127. Takahashi, K. and H.T. Hahn, Investigation of temperature dependency of electrical resistance changes for structural management of graphite/polymer composite. Journal of composite materials, 2011. 45(25): p. 2603-2611.

128. Davaji, B., et al., A patterned single layer graphene resistance temperature sensor. Scientific reports, 2017. 7(1): p. 1-10.

129. Kaidaorva, A., et al., Flexible, four-electrode conductivity cell for biologging applications. Results in Materials, 2019. 1: p. 100009.

130. Huang, Y., et al., Improved Performance of Flexible Graphene Heater Based on Repeated Laser Writing. IEEE Electron Device Letters, 2020. 41(3): p. 501-504.

131. He, M., et al., Laser-induced graphene enabled $1 D$ fiber electronics. Carbon, 2020. 168: p. 308-318.

132. Huang, L., et al., Self-Reporting and Photothermally Enhanced Rapid Bacterial Killing on a Laser-Induced Graphene Mask. ACS nano, 2020. 14(9): p. 12045-12053.

133. Yang, P. and X. Wang, COVID-19: a new challenge for human beings. Cellular \& molecular immunology, 2020. 17(5): p. 555-557.

134. Bird, R.B., Transport phenomena. Appl. Mech. Rev., 2002. 55(1): p. R1-R4.

135. Yang, L., et al., Novel gas sensing platform based on a stretchable laser-induced graphene pattern with self-heating capabilities. Journal of Materials Chemistry A, 2020. 8(14): p. 6487-6500.

136. Huang, L., et al., Laser-Induced Graphene: En Route to Smart Sensing. Nano-Micro Letters, 2020. 12(1): p. 1-17.

137. Wang, Z., et al., Highly sensitive flexible magnetic sensor based on anisotropic magnetoresistance effect. Advanced Materials, 2016. 28(42): p. 9370-9377.

138. Sagar, R.U.R., et al., Defect-induced, temperature-independent, tunable magnetoresistance of partially fluorinated graphene foam. Carbon, 2019. 143: p. 179-188.

139. Warren, S.G., R.E. Brandt, and T.C. Grenfell, Visible and nearultraviolet absorption spectrum of ice from transmission of solar radiation into snow. Applied optics, 2006. 45(21): p. 5320-5334. 
140. Naylon, A., Microwave resonant sensors. 2011, Cardiff University.

141. Xie, Y., et al., Rapid Synthesis of Zeolitic Imidazole Frameworks in Laser-Induced Graphene Microreactors. ChemSusChem, 2019. 12(2): p. 473479.

142. Lan, L., et al., One-step and large-scale fabrication of flexible and wearable humidity sensor based on laser-induced graphene for real-time tracking of plant transpiration at bio-interface. Biosensors and Bioelectronics, 2020: p. 112360.

143. Cai, J., et al., Laser direct writing of a highperformance all-graphene humidity sensor working in a novel sensing mode for portable electronics. ACS applied materials \& interfaces, 2018. 10(28): p. 23987-23996.

144. An, J., et al., All-graphene-based highly flexible noncontact electronic skin. ACS applied materials \& interfaces, 2017. 9(51): p. 44593-44601.

145. Vogel, J., et al., Activation energy for diffusion and welding of PLA films. Polymer Engineering \& Science, 2012. 52(8): p. 1693-1700.

146. Pawar, A.Y., et al., Terahertz technology and its applications. Drug invention today, 2013. 5(2): p. 157-163.

147. Shaikh, S.F. and M.M. Hussain, Multisensory graphene-skin for harsh-environment applications. Applied Physics Letters, 2020. 117(7): p. 074101.

148. Singh, S.P., et al., Laser-induced graphene layers and electrodes prevents microbial fouling and exerts antimicrobial action. ACS applied materials \& interfaces, 2017. 9(21): p. 18238-18247.

149. d'Amora, M., et al., Toxicity assessment of laserinduced graphene by zebrafish during development. Journal of Physics: Materials, 2020. 3(3): p. 034008.

150. Woerle, J. and H. Rost, Roll-to-roll production of transparent conductive films using metallic grids. MRS bulletin, 2011. 36(10): p. 789. 